\title{
TO IMPROVE THE ANALYSIS OF THE FINANCIAL SECURITY OF THE BUSINESS IN GEORGIAN ENTERPRISES
}

\section{IZOLDA CHILADZE}

Doctor of Economics,

Associate Professor

Ivane Javakhishvili Tbilisi State University, Georgia

izolda.chiladze@tsu.ge

Abstract. The purpose of this paper is to improve the analysis of the financial stability of enterprises and to identify the key factors affecting the establishment of the sustainable growth trend of enterprises (except the financial sector) in Georgia. Answering the question: what financial challenges do enterprises in Georgia face today and be designed consequently, recommendations for strengthening the financial stability of enterprises should be developed in Georgia, increasing solvency and mitigating bankruptcy risks? The necessity for the financial stability of enterprises is becoming increasingly important both for sustainable business growth and for the stability of the international financial market.

The subject of the study is the financial positions and financial results of the analytical enterprises. The first and second categories of joint-stock companies of Georgia and limited liability companies were selected as the research objects. Ten enterprises in total. The research methodology includes methods of economic and statistical analysis, factor analysis, vertical, horizontal, proportional and ratio analysis.

In order to improve financial management and control, the paper presents multi-factor models of several indicators created by the author. These are: the tree-factor model the coefficient of the organic structure of capital, an eight-factor model of profitability and a six-factor model of the Financial leverage.

The paper conducts practical research on the example of ten Georgian companies and concludes that the main challenges in todayss Georgia are the dangers of losing financial stability and bankruptcy. The reason for such a tense financial situation is not a "Corona-19».

According to the author, the main reasons for the instability of enterprises in Georgia are: The Irresponsibility of business owners and management, low management professionalism and hidden flows of cash resources.

KEYWORDS: FINANCIAL STABILITY, SUSTAINABLE GROWTH, SOLVENCY, PROFITABILITY, FINANCIAL RISKS.

For citation: Chiladze I. (2020). To Improve of Analysis of the Financial Security of the Business in Georgian Enterprises. Globalization and Business, №9, pp. 135-147 (In Georgian). https://doi.org/10.35945/gb.2020.09.017 


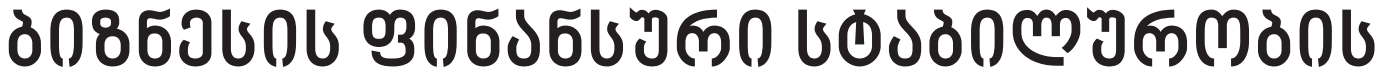

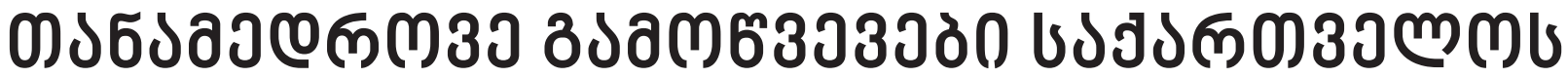

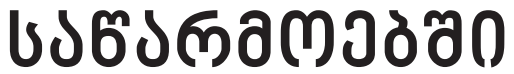

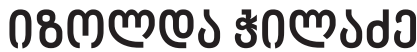

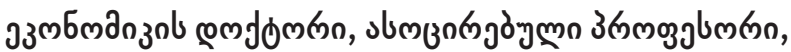

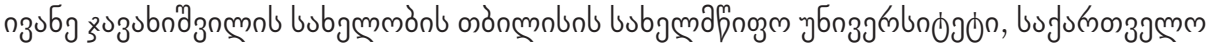
izolda.chiladze@tsu.ge

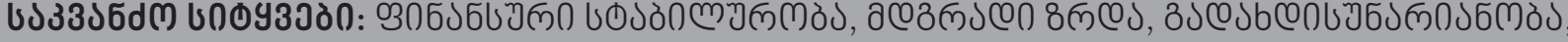

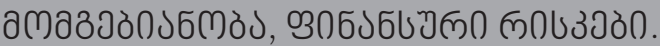

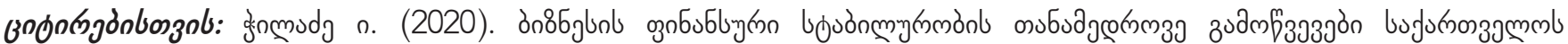

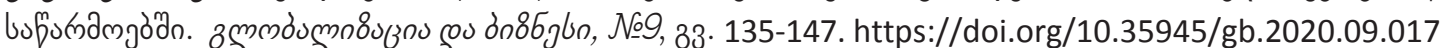

\section{ฆว৮১3১ल0}

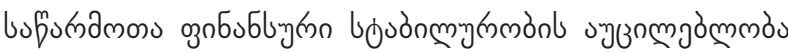

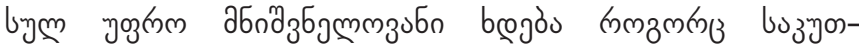

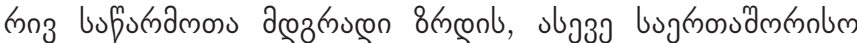

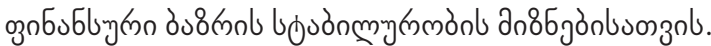

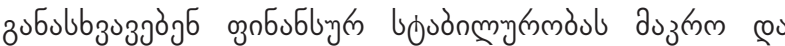

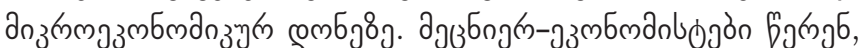

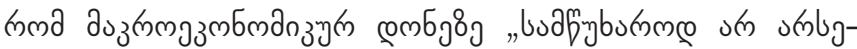

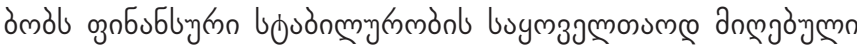

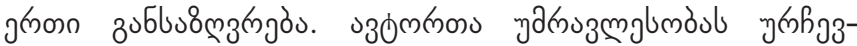

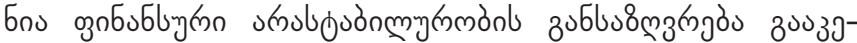

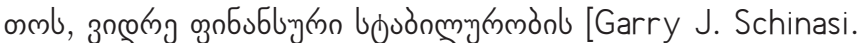

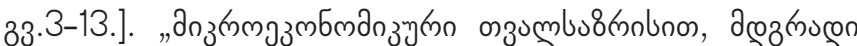

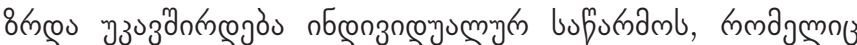
cougुydб

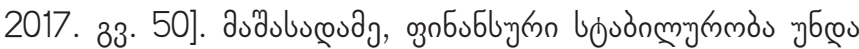

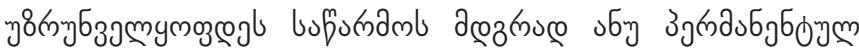

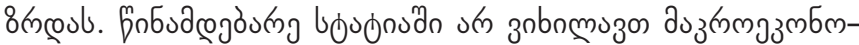

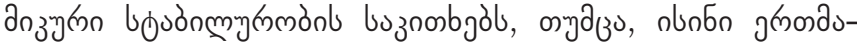

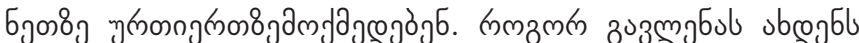

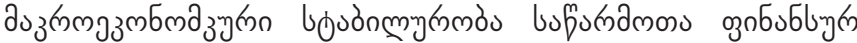

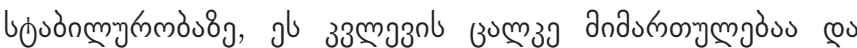

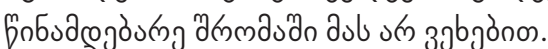

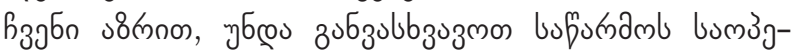

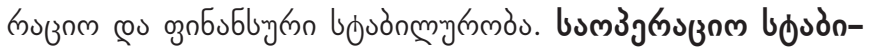

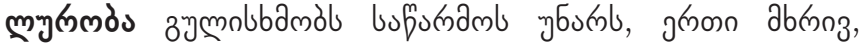

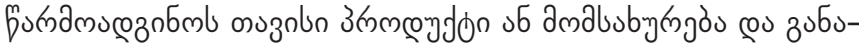

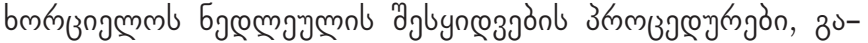

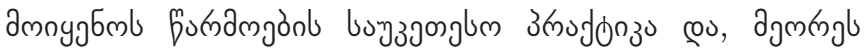

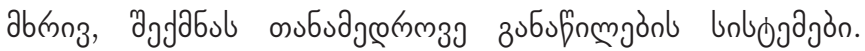

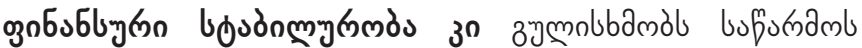

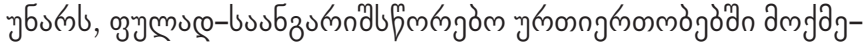

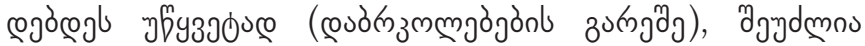

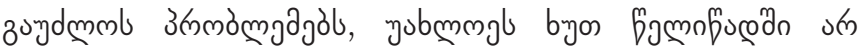

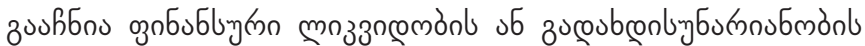

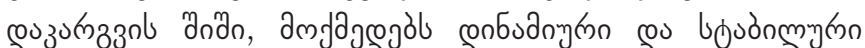

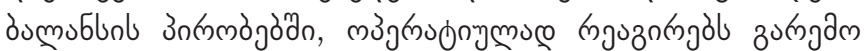

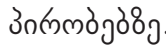

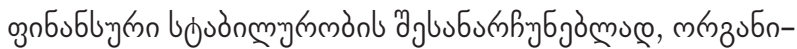

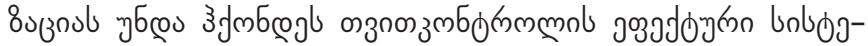
du, euogyd

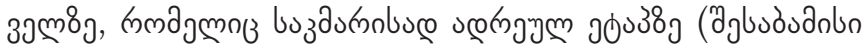

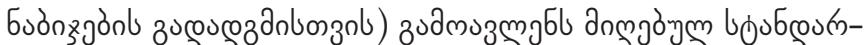

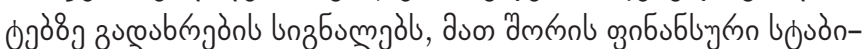

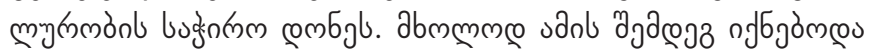

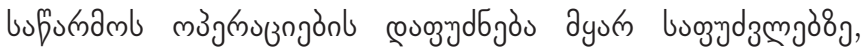

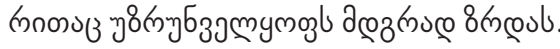

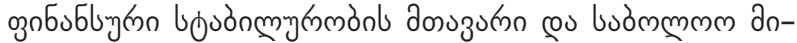

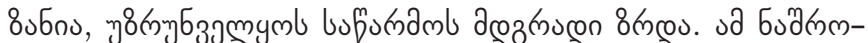

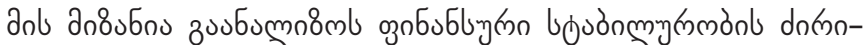

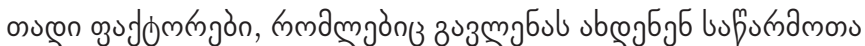

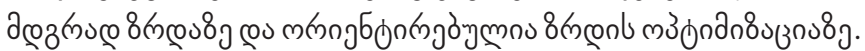

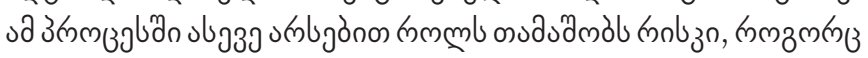

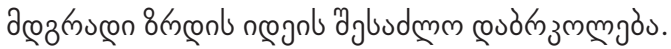

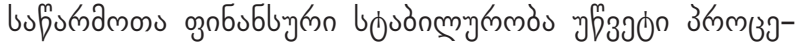

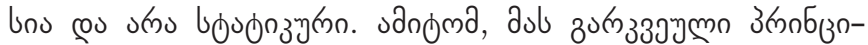

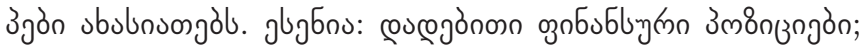




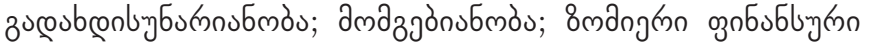

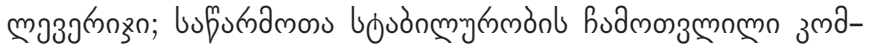

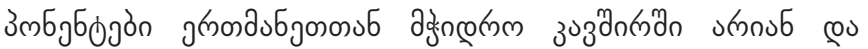

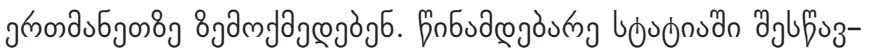

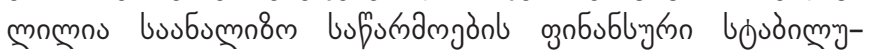

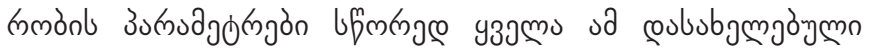

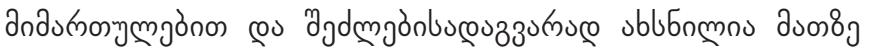

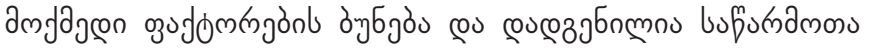

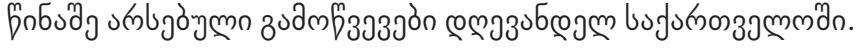

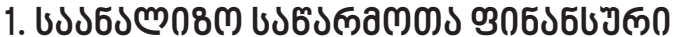

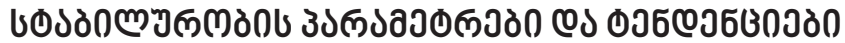

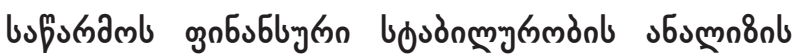

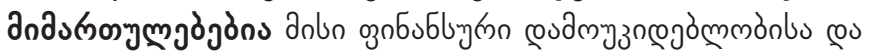

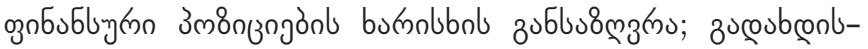

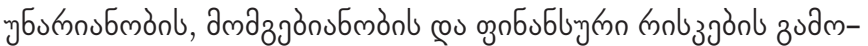
उзм9з3.

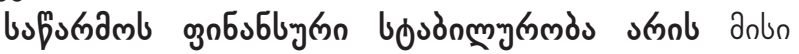

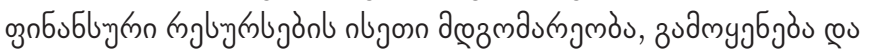

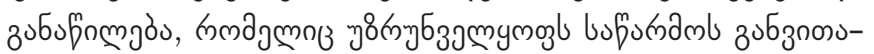

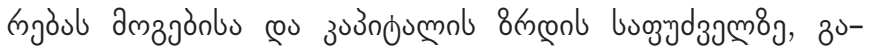

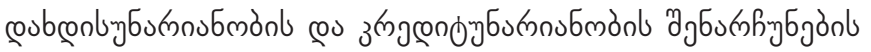
उnफलòjòn.

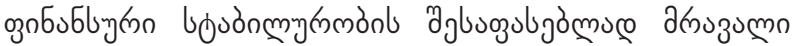

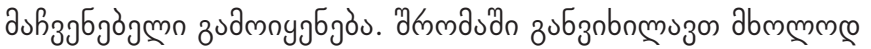
dosuzum du

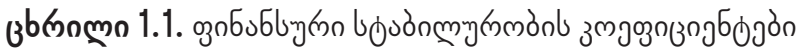
2017-2018 f6

\begin{tabular}{|c|c|c|c|c|c|c|}
\hline \multirow[b]{2}{*}{ 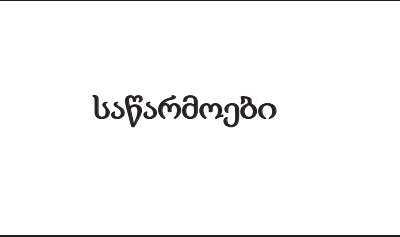 } & \multicolumn{3}{|c|}{2017} & \multicolumn{3}{|c|}{2018} \\
\hline & 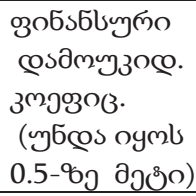 & 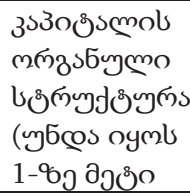 & 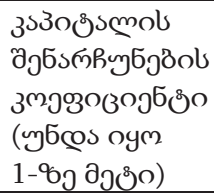 & 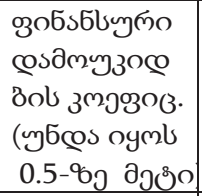 & 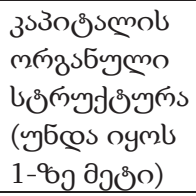 & 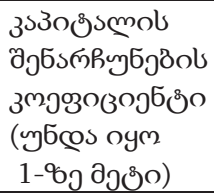 \\
\hline 1. bl " $3^{\mathrm{m}} \mathrm{z}^{\mathrm{s}} 3^{\mathrm{m}} \mathrm{mss}^{\prime \prime}$ & $0.40^{*}$ & $0.667^{*}$ & 1.07 & $0.44^{*}$ & $0.794^{*}$ & 1.22 \\
\hline 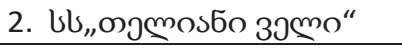 & 0.85 & 5.689 & 2.18 & 0.84 & 5.11 & 1.10 \\
\hline 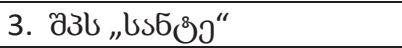 & 0,79 & 3.751 & 1.21 & 0.83 & 4.79 & 1.13 \\
\hline 4. bl , ,бозмп s“ & $0,17^{*}$ & $0.621 *$ & $0,37^{*}$ & 0.56 & 1.30 & 1.01 \\
\hline 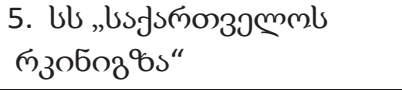 & $0.42 *$ & $0.77^{*}$ & $0.76^{*}$ & $0.23^{*}$ & $0.31 *$ & $0.43^{*}$ \\
\hline 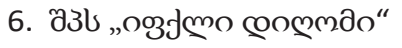 & 0,85 & 5.68 & $0.96^{*}$ & 0.73 & 2.65 & 1.09 \\
\hline 7. اb „вэщзомо“ & $(0.61)^{*}$ & $(0.49)^{*}$ & $0.99 *$ & $0 *$ & $(0.49)^{*}$ & $0 *$ \\
\hline 8. ub „,ைলூslıo“ & $0.48^{*}$ & $0.92 *$ & 1.12 & 0.53 & 1.13 & 1.18 \\
\hline 9. ৮ا „одуœо L“ & $0.21^{*}$ & $0.26^{*}$ & $0.80^{*}$ & $0.36^{*}$ & $0.57^{*}$ & 2.22 \\
\hline 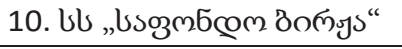 & 0,86 & 29.4 & $0,96 *$ & 0.94 & 16.36 & $0.69^{*}$ \\
\hline
\end{tabular}

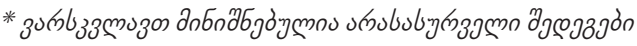

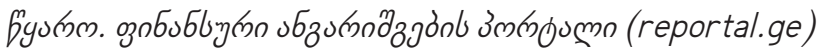

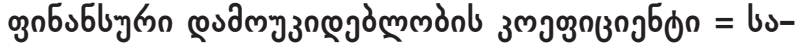

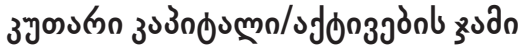

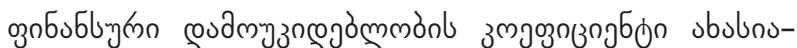

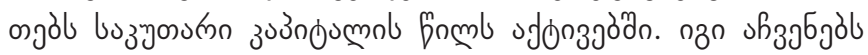

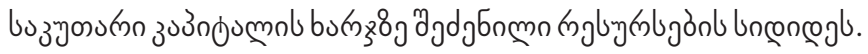

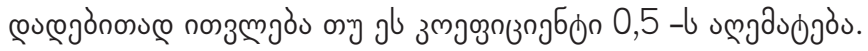

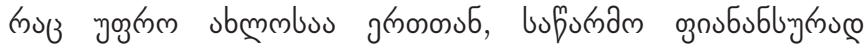

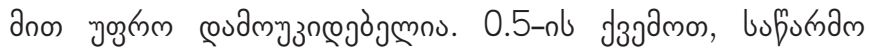

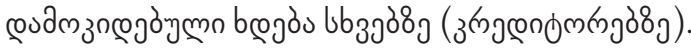

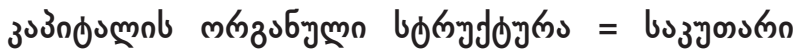

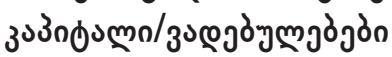

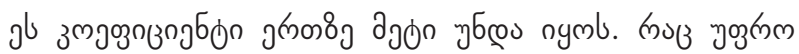

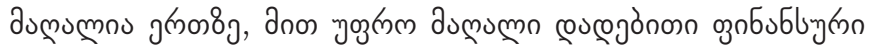

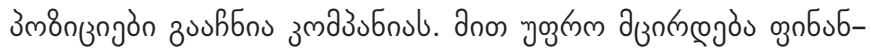
bygno moluzgon

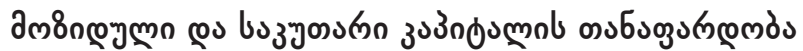
= z०m

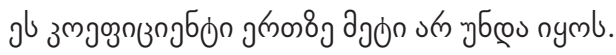

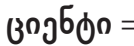

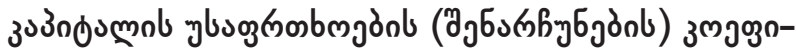

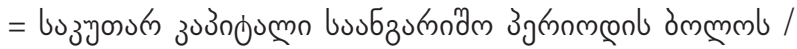

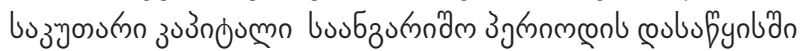




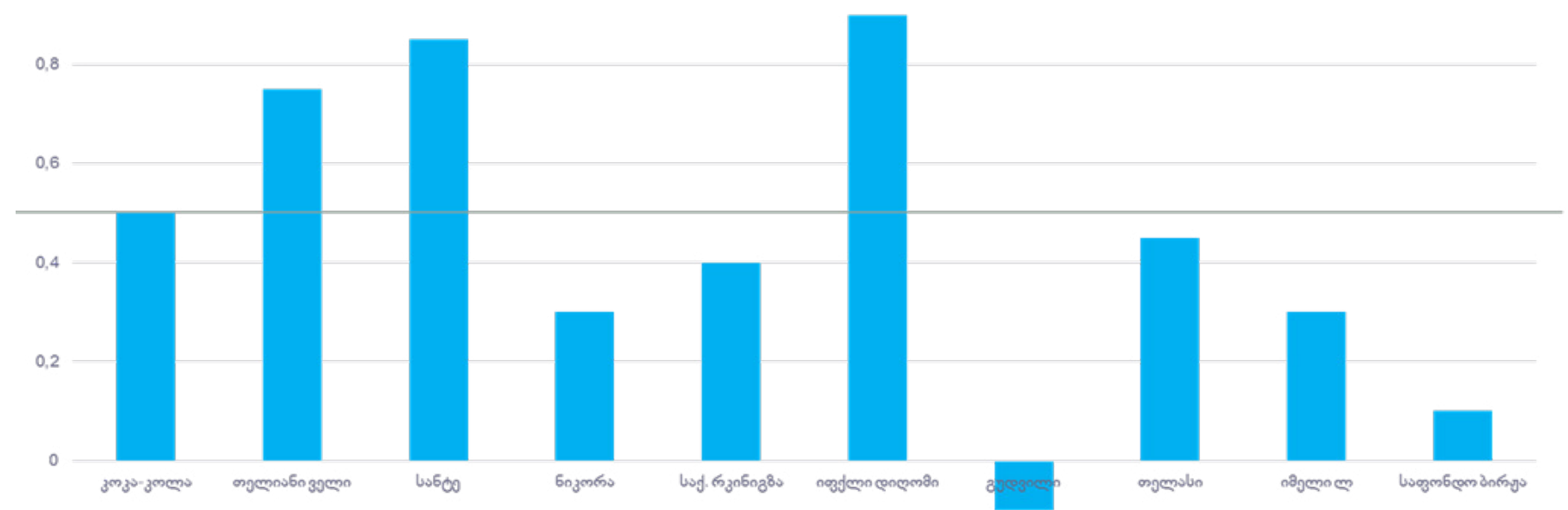

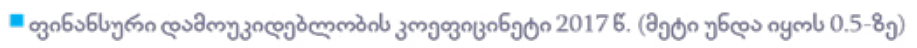

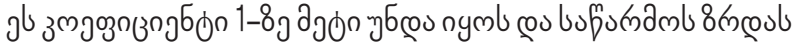

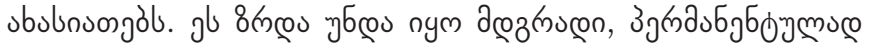

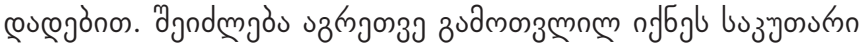

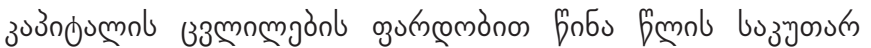
उ०3กбumosob.

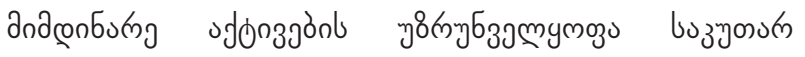

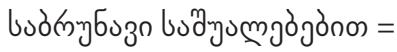

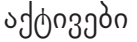

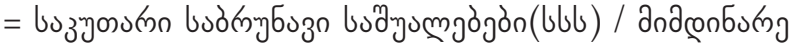

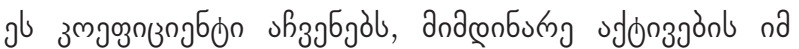

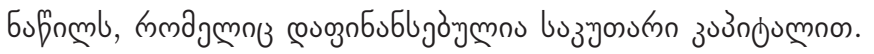
anln nбogrisumn bu

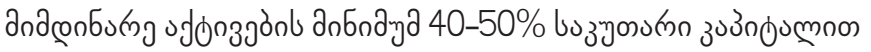

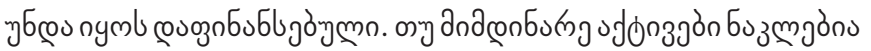

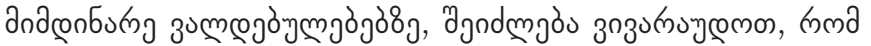

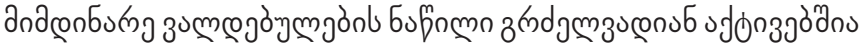

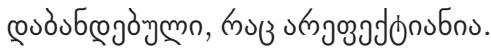

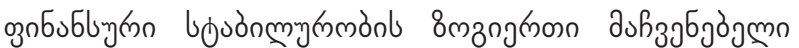

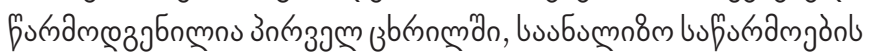
aงzumnosog.

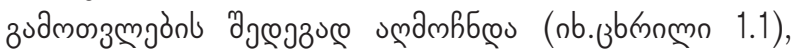

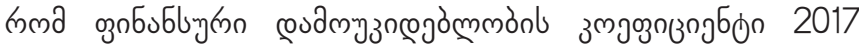

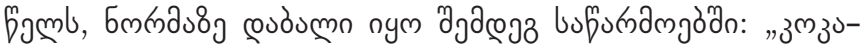

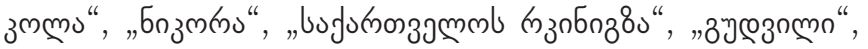

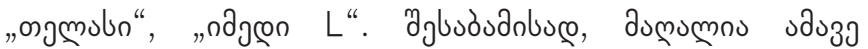

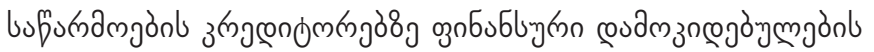

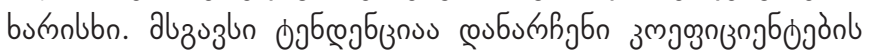

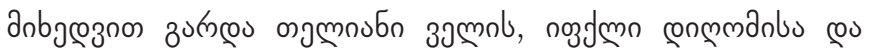

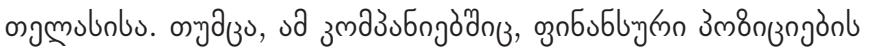

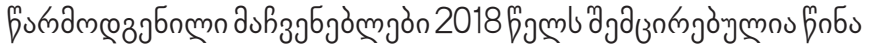

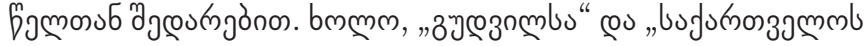

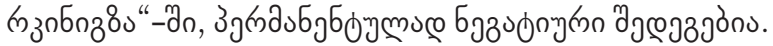

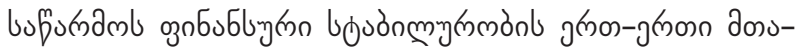

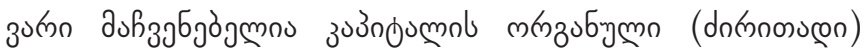

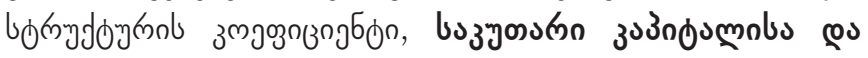

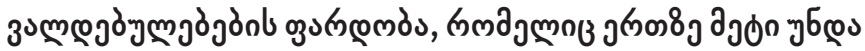

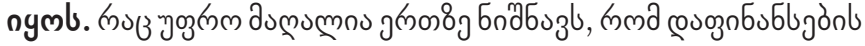

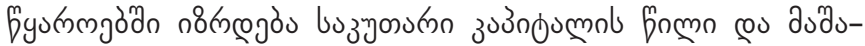

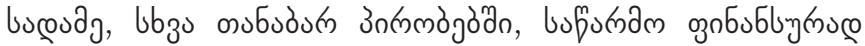

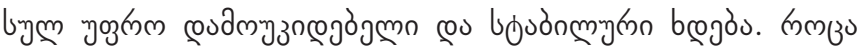

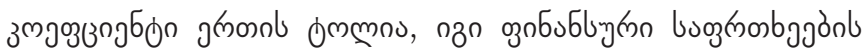

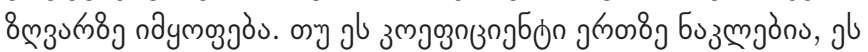

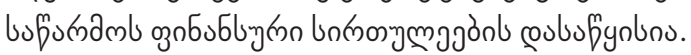

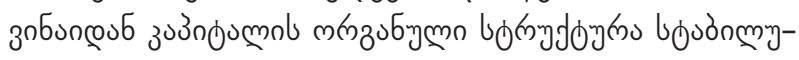

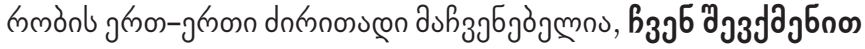

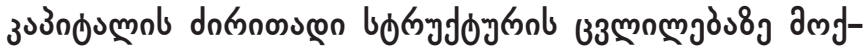

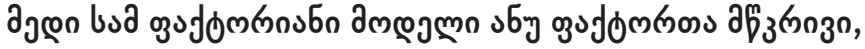

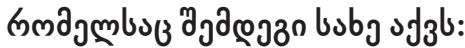

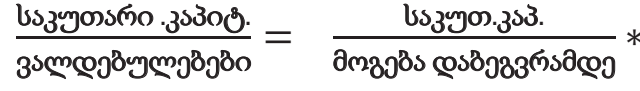

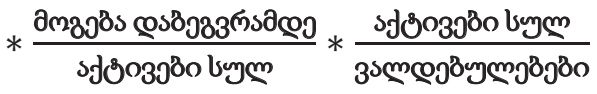

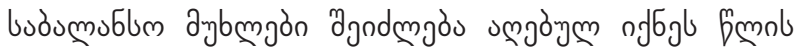

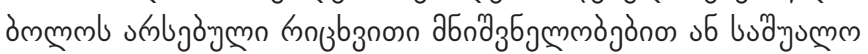

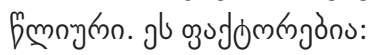

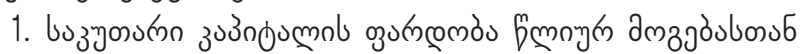

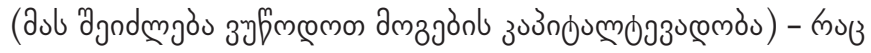

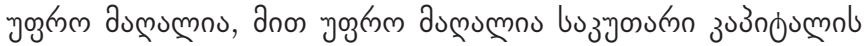




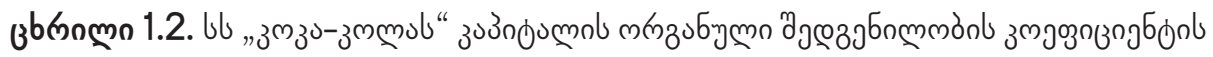

\begin{tabular}{|c|c|c|c|c|c|}
\hline \multirow[b]{2}{*}{ 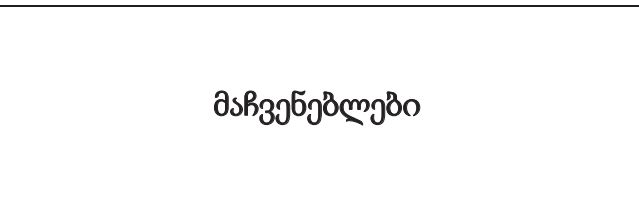 } & \multirow[b]{2}{*}{2016} & \multicolumn{2}{|c|}{2017} & \multicolumn{2}{|c|}{2018} \\
\hline & & зल्ग\%. & 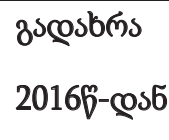 & зㅆㅇ․ & $\begin{array}{l}\text { 3scosbms } \\
20177^{-} \\
\text {cos6 }\end{array}$ \\
\hline 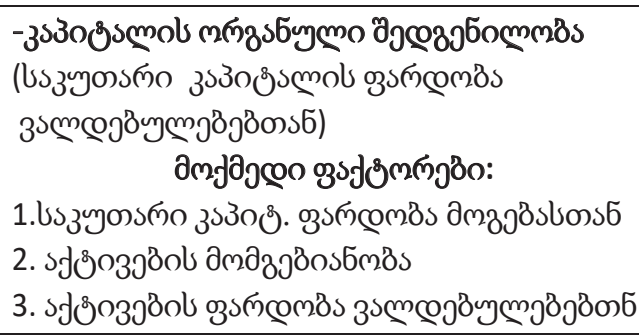 & $\begin{array}{l}4.4475 \\
0.0912 \\
1.4841\end{array}$ & $\begin{array}{l}12.2028 \\
0.0329 \\
1.6614\end{array}$ & $\begin{array}{l}+7.7553 \\
-0.0583 \\
+0.1773\end{array}$ & $\begin{array}{l}5.6147 \\
0.0788 \\
1.7944\end{array}$ & $\begin{array}{l}-6.5881 \\
+0.0459 \\
+0.133\end{array}$ \\
\hline 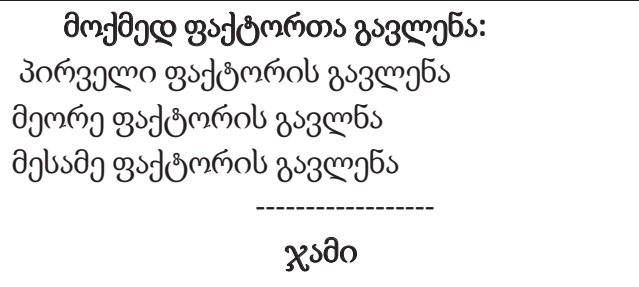 & & & $\begin{array}{l}+1.0497 \\
-1.0558 \\
+0.0711 \\
+0.065\end{array}$ & & $\begin{array}{r}-6.5881 \\
+0.0459 \\
+0.0588 \\
-0 .- \\
+0.1269\end{array}$ \\
\hline
\end{tabular}

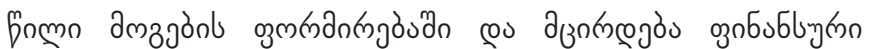
molizgòn;

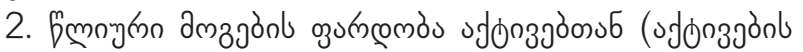

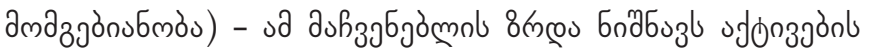

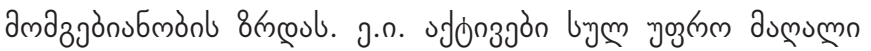

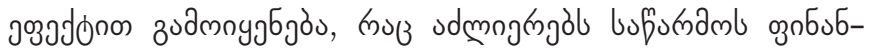

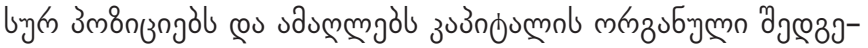
Enmmònl bumnlbl.

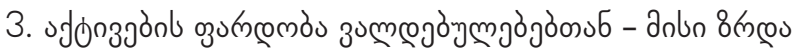

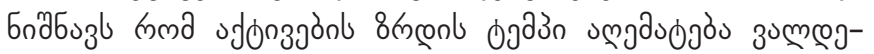

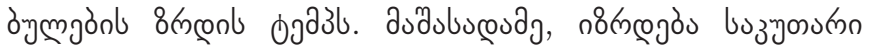
zuउn бu

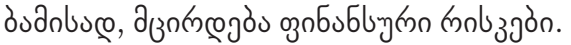

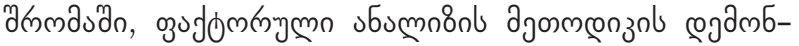

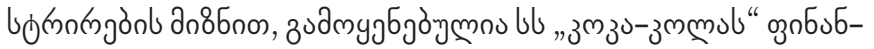

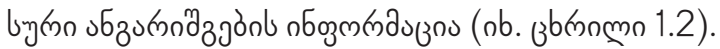

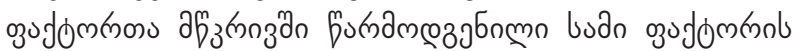

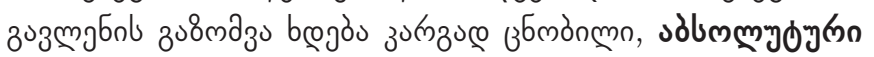

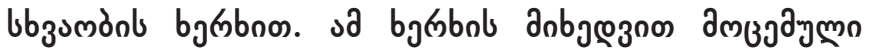

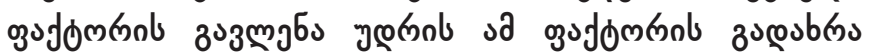

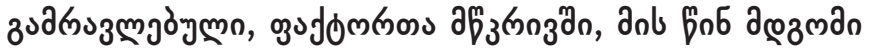

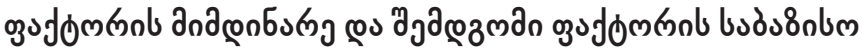

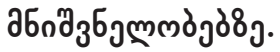

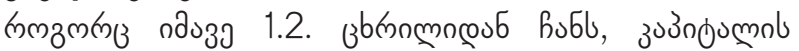

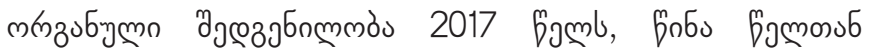

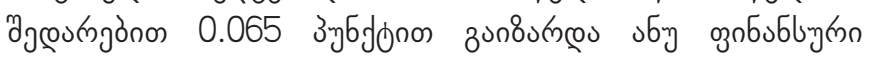

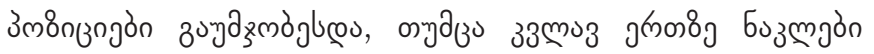

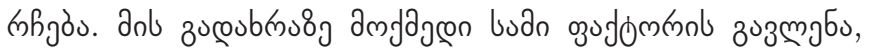

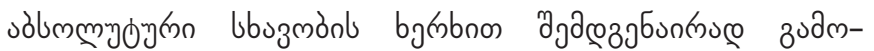
nos3mgos:

$$
\begin{aligned}
& (+7.7553) * 0.0912 * 1.4841=+1.0497 \\
& 12.2028 *(-0.0583) * 1.4841=-1.0558 \\
& 12.2028 * 0.0329 *(+0.1773)=+0.0711
\end{aligned}
$$

$\xi 302 n+0.065$

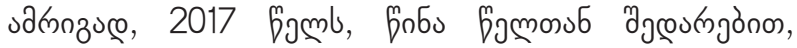
उ०उnou

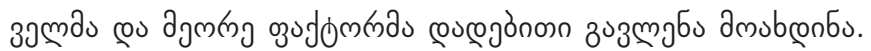

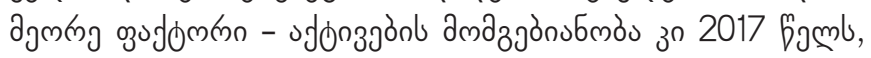

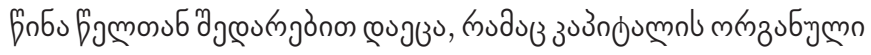

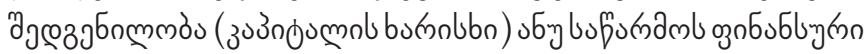

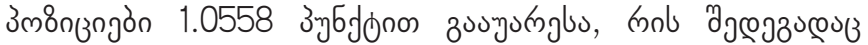

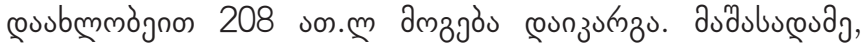

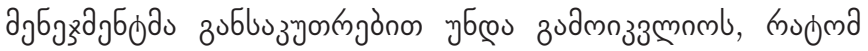

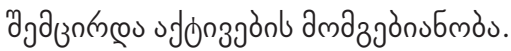

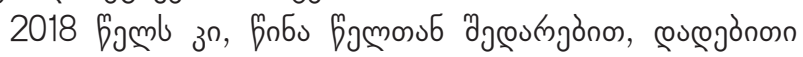

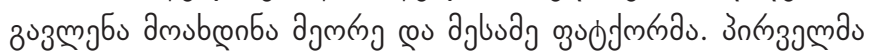

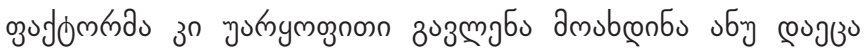

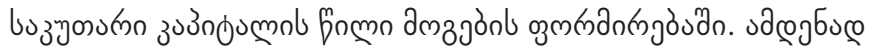

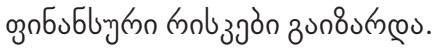

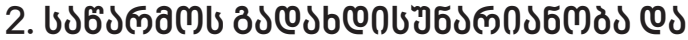

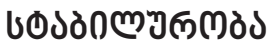

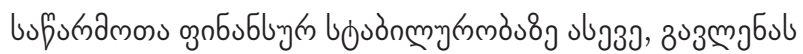

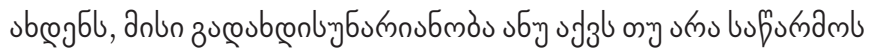

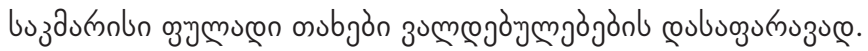

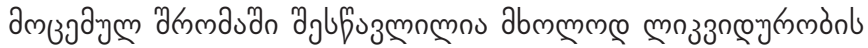

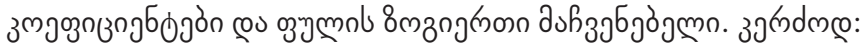




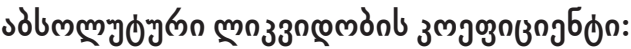

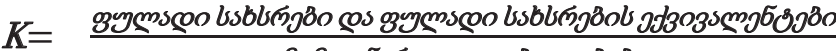

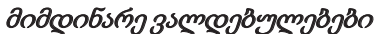

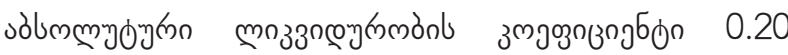

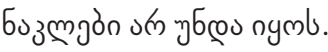

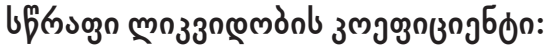

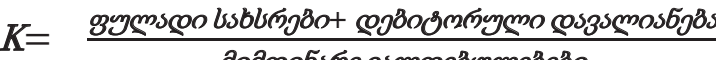

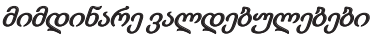

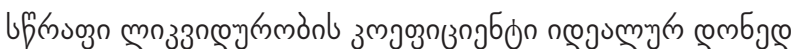

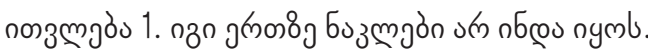

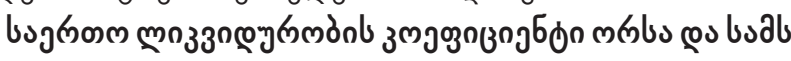

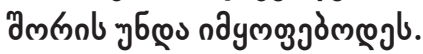

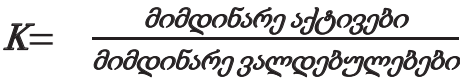

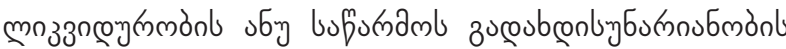

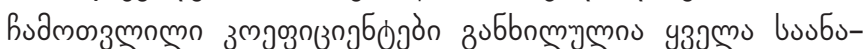

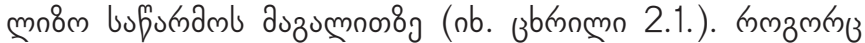

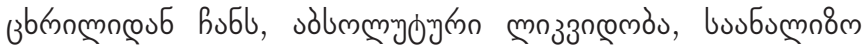

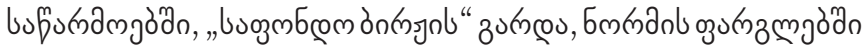

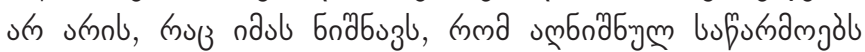

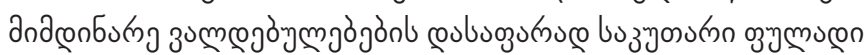

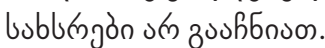

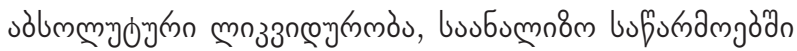

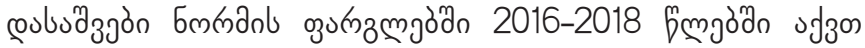

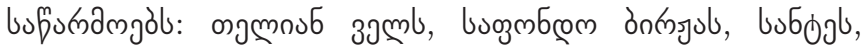

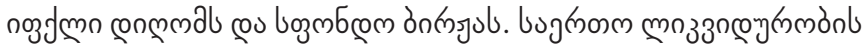

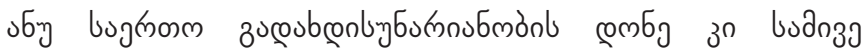

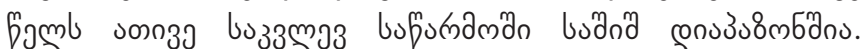

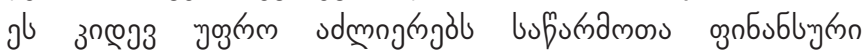

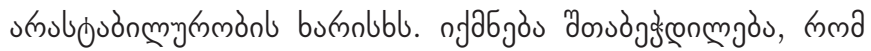

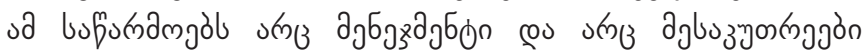

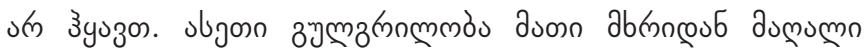

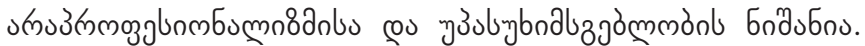

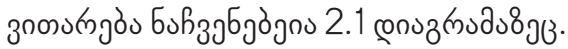

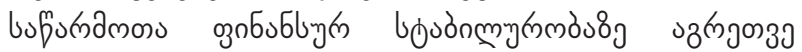
зงзмgб

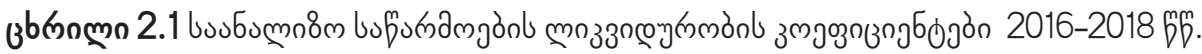

\begin{tabular}{|c|c|c|c|c|c|c|}
\hline \multirow[t]{2}{*}{ 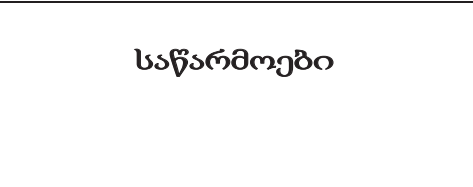 } & \multicolumn{3}{|c|}{ 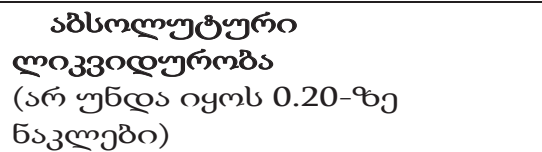 } & \multicolumn{3}{|c|}{ 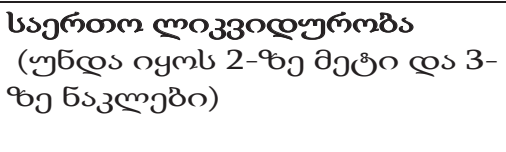 } \\
\hline & 2016 & 2017 & 2018 & 2016 & 2017 & 2018 \\
\hline ul $3_{\mathrm{m}} \mathrm{z}_{\mathrm{s}}-3_{\mathrm{m}} \mathrm{ms}$ & $0,0289 *$ & $0,0605^{*}$ & $0.003^{*}$ & $0,4428 *$ & $0,6564^{*}$ & $0.77^{*}$ \\
\hline 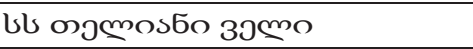 & 0,6789 & 0,7260 & 0.47 & 2,6291 & 2,4648 & $0.99^{*}$ \\
\hline 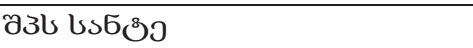 & 0,3870 & 0,3923 & 0.25 & $1,7237 *$ & $1,5820 *$ & $1.84^{*}$ \\
\hline ub Бозलळы & $0,0405 *$ & $0,1466^{*}$ & $0.13^{*}$ & $0,5238 *$ & $0,5630 *$ & $0.34^{*}$ \\
\hline ub bsf১ল & 1,2709 & $1,0718^{*}$ & $0.0004^{*}$ & $1,8904^{*}$ & $1,4326^{*}$ & $0.91 *$ \\
\hline 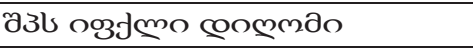 & 0,7566 & 0,1770 * & 2.40 & $6,2382 *$ & 2,0368 & $6.13^{*}$ \\
\hline Ul зумезомо & $0,0982 *$ & $0,1216^{*}$ & $0.10^{*}$ & $0,8706 *$ & $0,7348^{*}$ & $0.70^{*}$ \\
\hline ub ojmslo & $0,1805 *$ & $0,1501^{*}$ & $0.07^{*}$ & $0,7233 *$ & $0,6705^{*}$ & $0.63^{*}$ \\
\hline 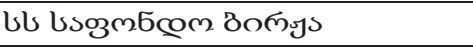 & 4,7507 & 4,9249 & 3.46 & $5,1618^{*}$ & $5,4033^{*}$ & $6.83^{*}$ \\
\hline ul odjœ๐ L & $0,0908 *$ & $0,0369^{*}$ & $0.06^{*}$ & $0,8694^{*}$ & $0,8433 *$ & $1.36^{*}$ \\
\hline
\end{tabular}

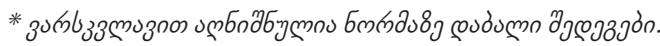

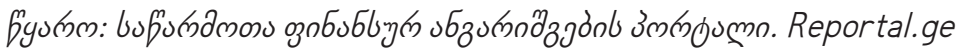

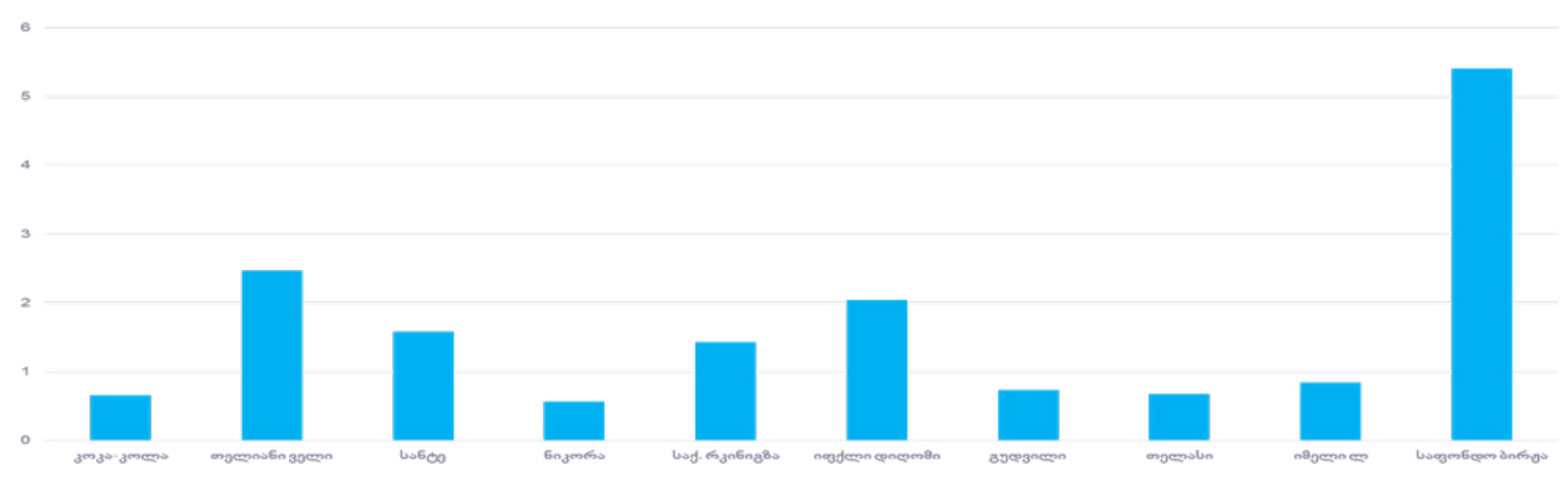

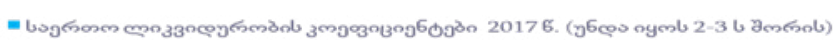

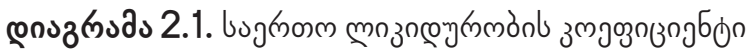




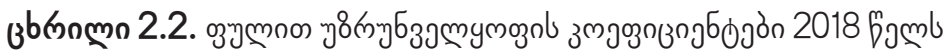

\begin{tabular}{|c|c|c|c|c|}
\hline иsf̧sпдмgд̈о & 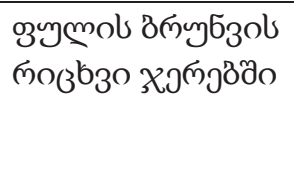 & 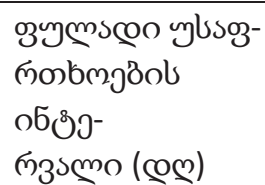 & 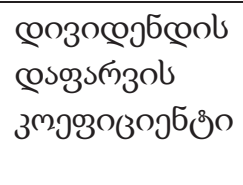 & 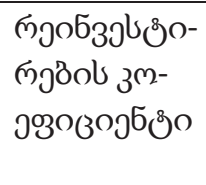 \\
\hline 1. bl ",3м.зs-zмms" & 144.41 & 5.03 & 4.56 & $-0.003^{*}$ \\
\hline 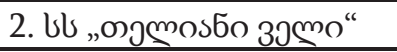 & 5.59 & 97.4 & 0.60 & 0.39 \\
\hline 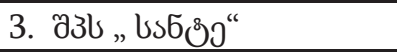 & 20.76 & 15.76 & 1 & 0.51 \\
\hline 4. bl „бозмпь“ & 163.5 & 0.04 & 1.56 & $-0.001^{*}$ \\
\hline 5. ub „,usf. mзобоз & 1.75 & 135.6 & 1 & $-1.28^{*}$ \\
\hline 6. bl „дуюзомо“ & 35.87 & 8.01 & 1.07 & $-3.29 *$ \\
\hline 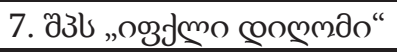 & 153.22 & 31.77 & 1 & $-6.02 *$ \\
\hline 8. ub „,лmsbа“ & 37.16 & 7.83 & 1.09 & $-0.34^{*}$ \\
\hline 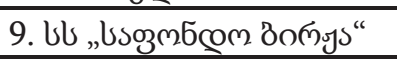 & 0.55 & 423 & 0 & $-1.61^{*}$ \\
\hline 10. ৬b „одэœо L“ & 36.39 & 13.77 & 1.14 & 0.12 \\
\hline
\end{tabular}

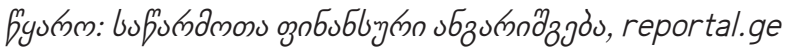

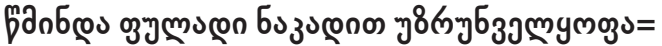

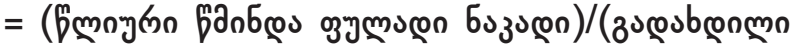

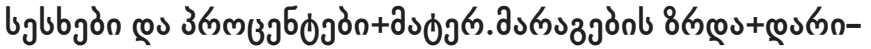

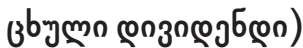

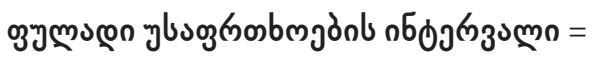

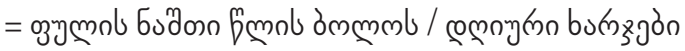

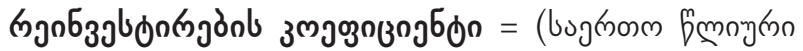

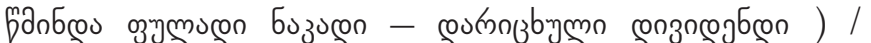

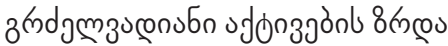

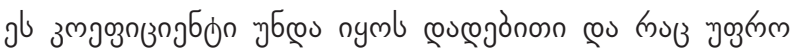

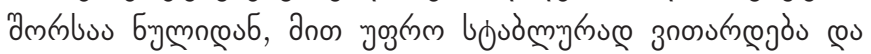

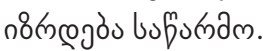

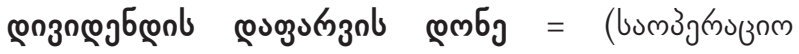

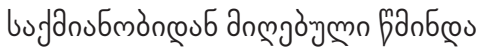

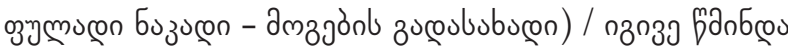

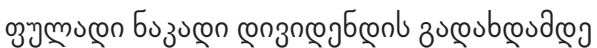

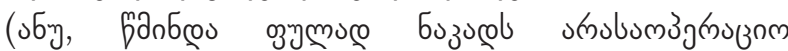

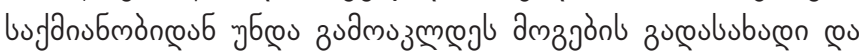

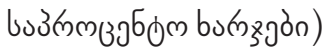

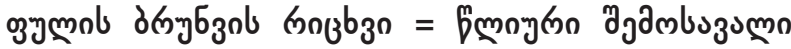

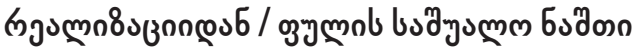

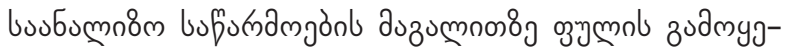

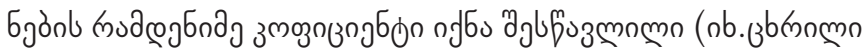

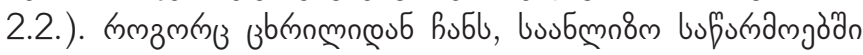

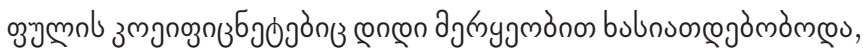

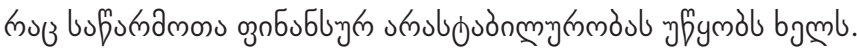

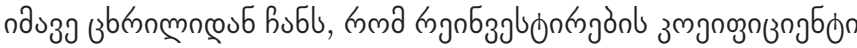

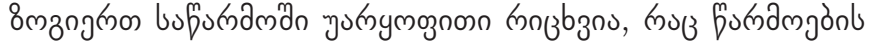

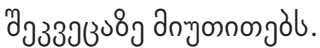

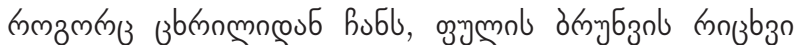

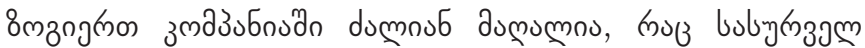

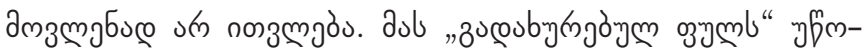

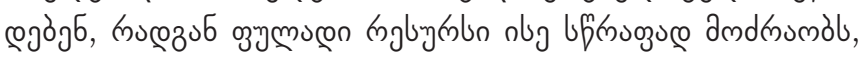

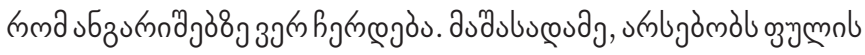

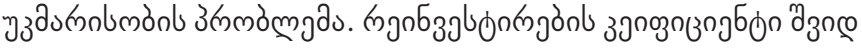

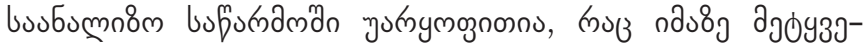

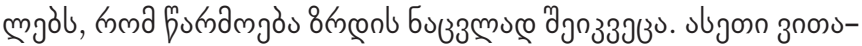

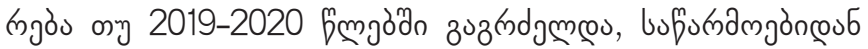

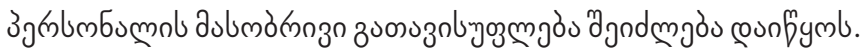

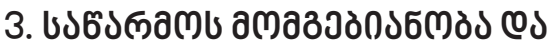

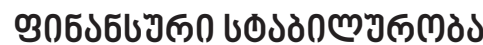

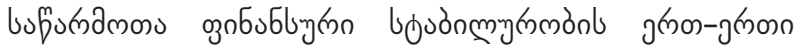
абпаз

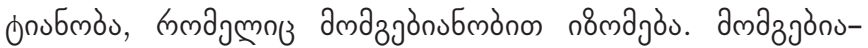

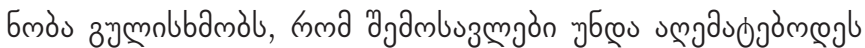

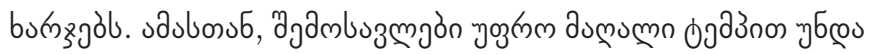

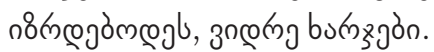

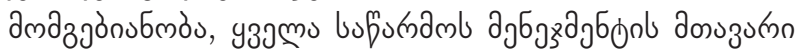

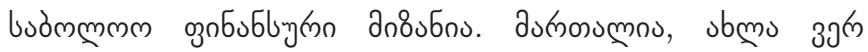

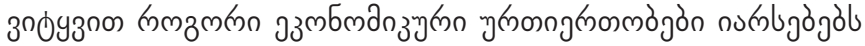

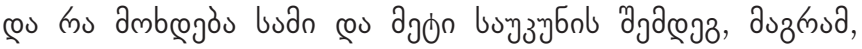

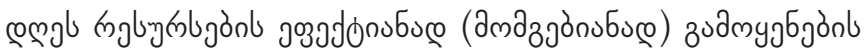

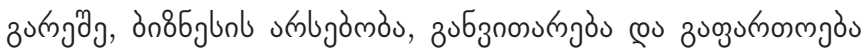

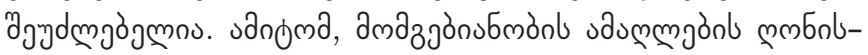

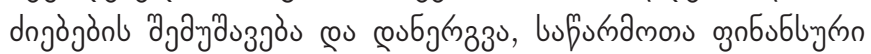

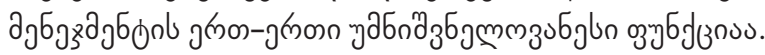

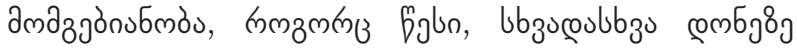




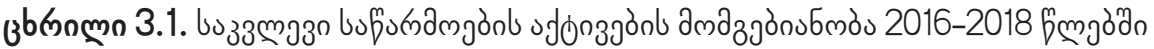

\begin{tabular}{|c|c|c|c|}
\hline \multirow[t]{2}{*}{ 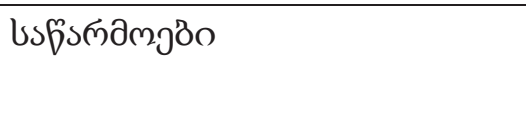 } & \multicolumn{3}{|l|}{ 6mjoo } \\
\hline & 2016 & 2017 & 2018 \\
\hline 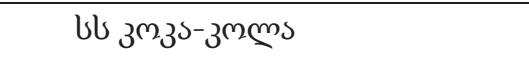 & $(0.0027)$ & 0.0329 & 0.0828 \\
\hline 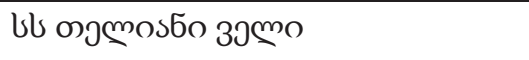 & 0.0409 & 0.0739 & 0.0339 \\
\hline aろb bs680 & - & 0.1497 & 0.1028 \\
\hline bl бозмпs & $(0.095)$ & 0.044 & 0.1589 \\
\hline 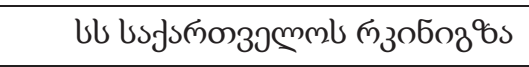 & & $(0.1247)$ & $(0.2516)$ \\
\hline 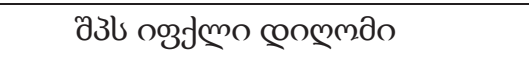 & 0.7218 & 0.5472 & 0.4745 \\
\hline вэусзомо & - & 0.0041 & 0.0725 \\
\hline ub oлmsıb & 0.1234 & 0.0804 & 0.0835 \\
\hline usogmberm domass & $(0.0772)$ & $(0.0349)$ & $(0.1541)$ \\
\hline одјœо m & $(0.0764)$ & $(0.0449)$ & 0.0607 \\
\hline
\end{tabular}

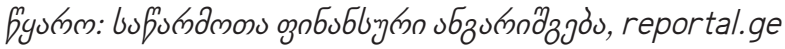

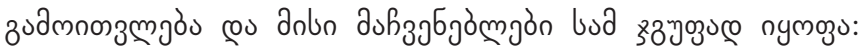

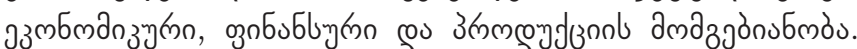

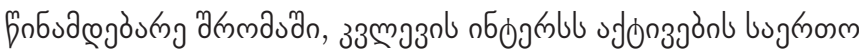

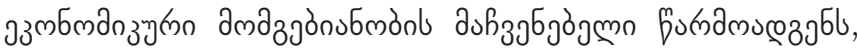

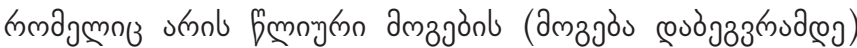

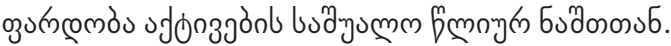

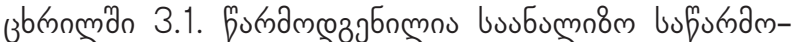

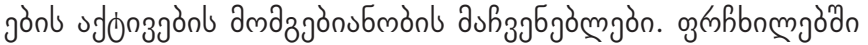

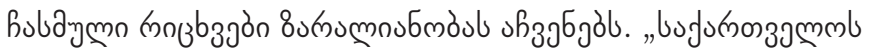

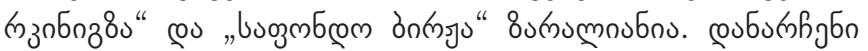

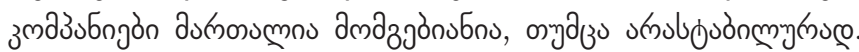

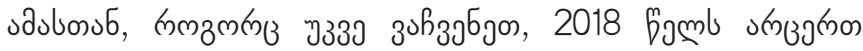

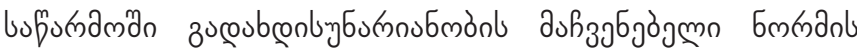

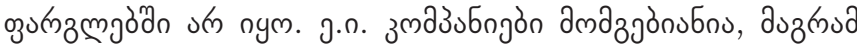

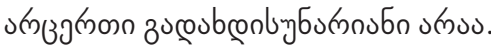

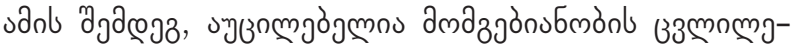

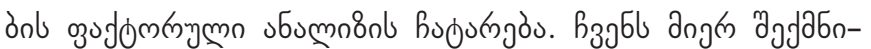

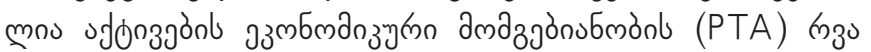

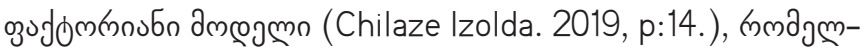
bos ggạgan bubg odst:

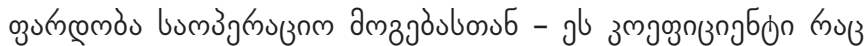

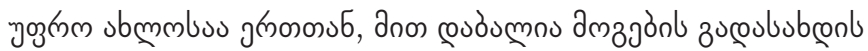

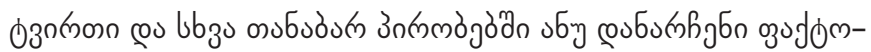

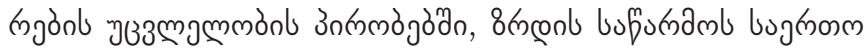

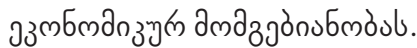

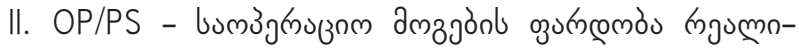

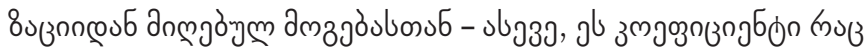

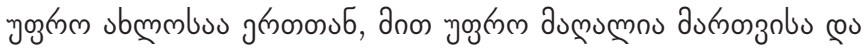

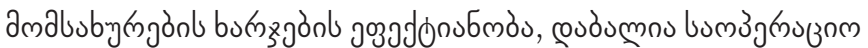

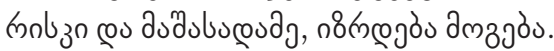

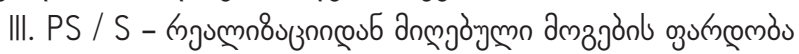

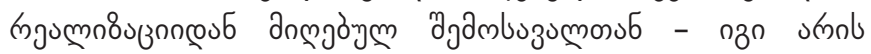

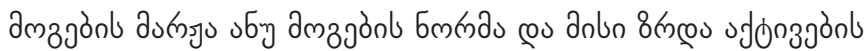

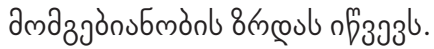

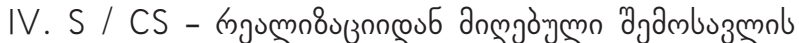

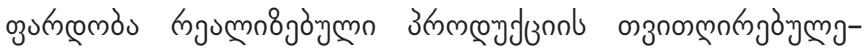

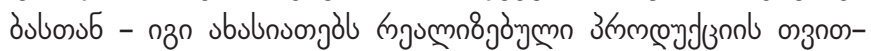

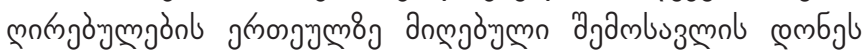

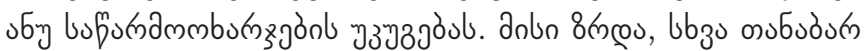

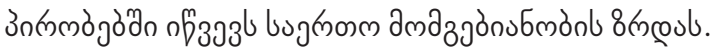

$$
P T A=\frac{P}{T A}=\frac{P}{O P} * \frac{O P}{P S} * \frac{P S}{S} * \frac{S}{C S} * \frac{C S}{O E x} * \frac{O E x}{(T A+C A)} * \frac{(T A+C A)}{O w \cdot C} * \frac{O w \cdot C}{T A}
$$

\section{bogob,}

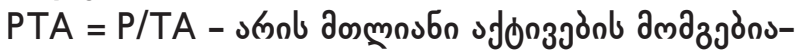

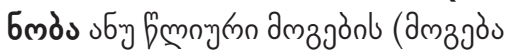

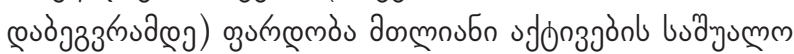

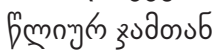

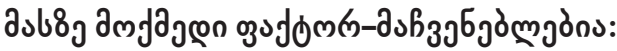

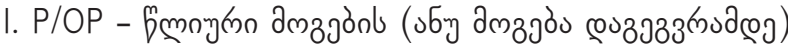

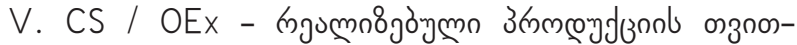

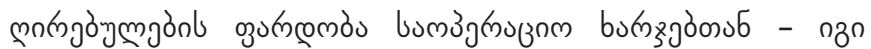

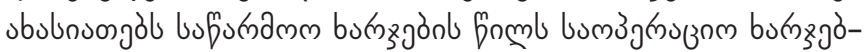

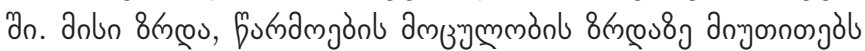

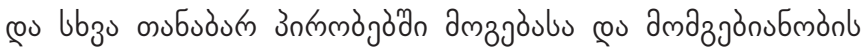

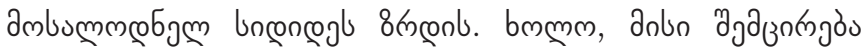

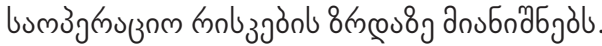




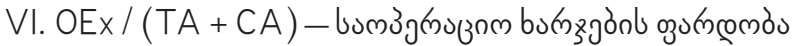

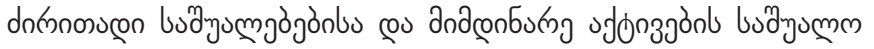

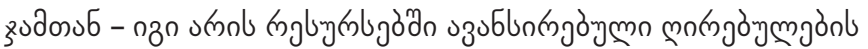

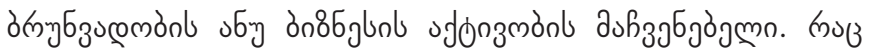

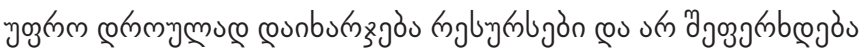

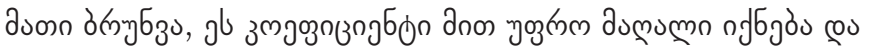

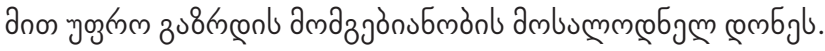

VII. (TA + CA ) / Ow.C - dnmnonsenn budyum göjònbs pou

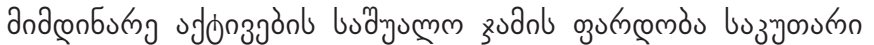

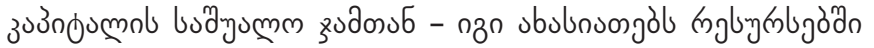

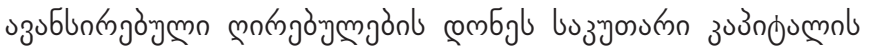

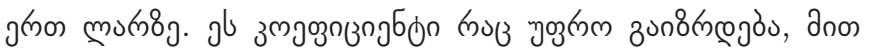

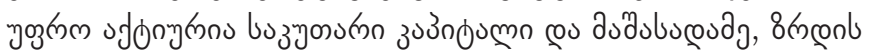

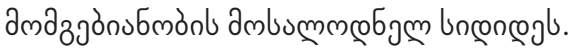

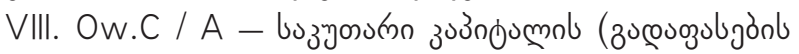

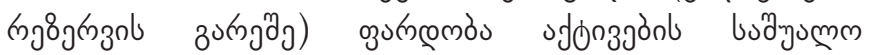

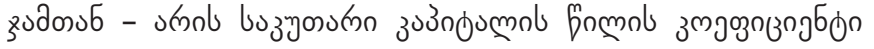

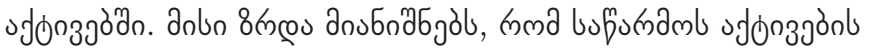

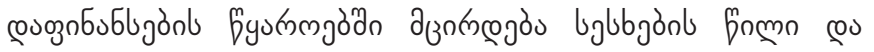

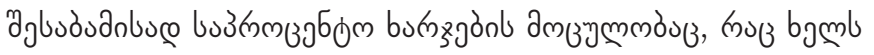

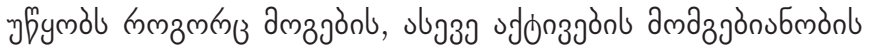
combols 8 monol.

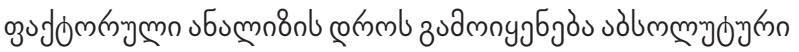

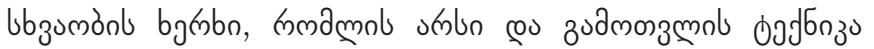

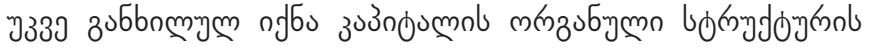

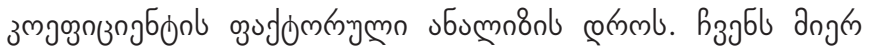

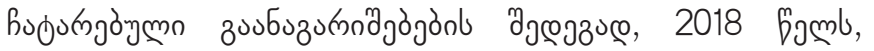

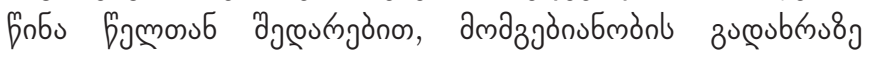

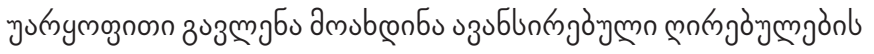

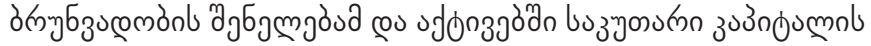

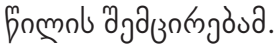

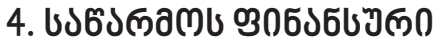

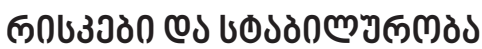

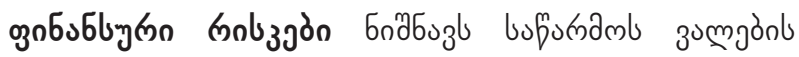

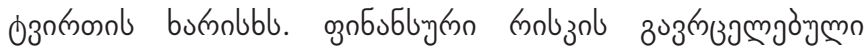

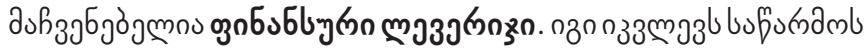

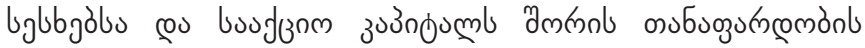

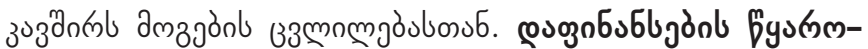

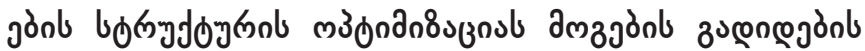

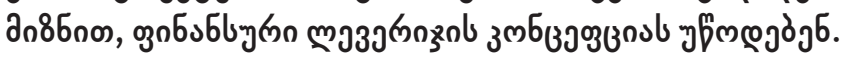

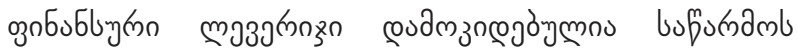

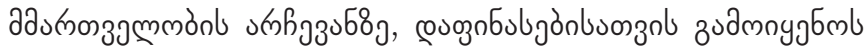

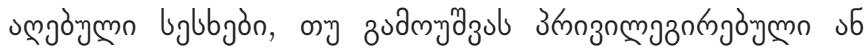

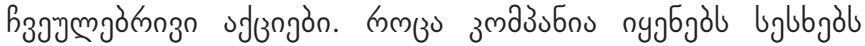

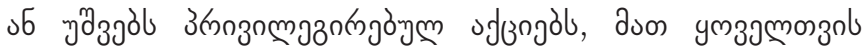

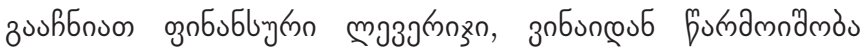

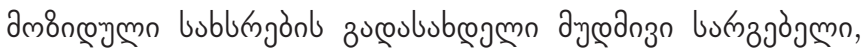

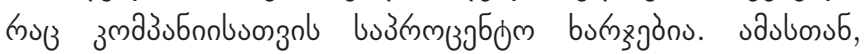

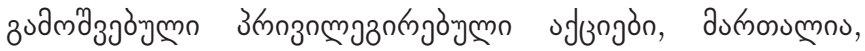

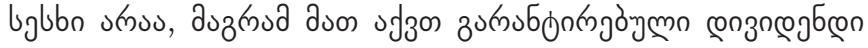

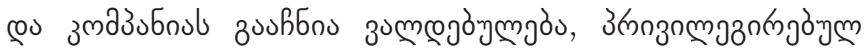

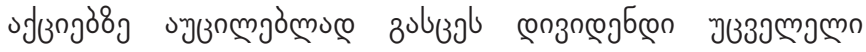

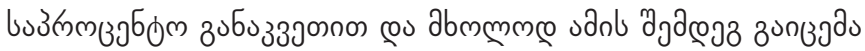

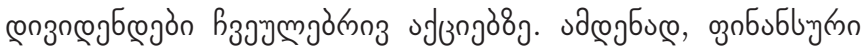

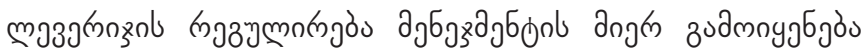

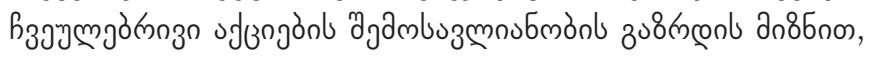

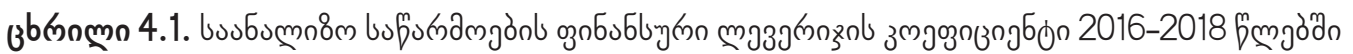

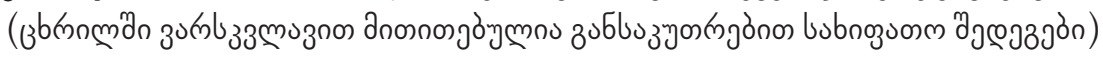

\begin{tabular}{|c|c|c|c|c|c|c|c|c|}
\hline \multirow[t]{2}{*}{3 м д 3 s 6 о э } & \multicolumn{3}{|c|}{ 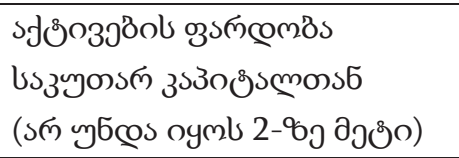 } & \multicolumn{3}{|c|}{ 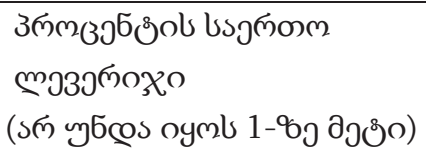 } & \multicolumn{2}{|c|}{ 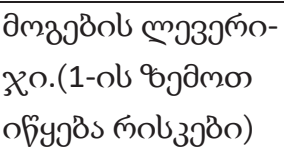 } \\
\hline & 2016 & 2017 & 2018 & 2016 & 2017 & 2018 & 2017 & 2018 \\
\hline 1. ub $3^{\mathrm{m}} 3^{s-} 3^{\mathrm{m}} \mathrm{ms}$ & 2.663 & 2.499 & 2.142 & 1.202 & 0.823 & 0.327 & 2.90 & 1.36 \\
\hline 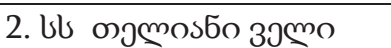 & 1.372 & 1.176 & 1.196 & 0.326 & 0.176 & 0.204 & 0.95 & 1.36 \\
\hline 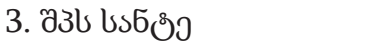 & 1,223 & 1.268 & 1.207 & -0.336 & 0.008 & 0.062 & 0.95 & 0.96 \\
\hline 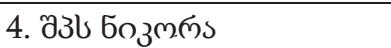 & 13.23 & 5.963 & 1.787 & 5.804 & 0.451 & 0.493 & 0,78 & 0.61 \\
\hline 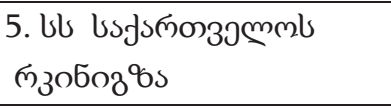 & 2.017 & 2.71 & 4.278 & 0.986 & -0.356 & -0.1318 & $(0.79)^{*}$ & $(0.90)^{*}$ \\
\hline 6. ub зуязомо & $(1,47)^{*}$ & $(34.5)$ & $(1.023) *$ & - & $(1.449) *$ & 0.159 & 0.99 & $1.18^{*}$ \\
\hline 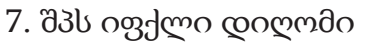 & 1.061 & 1.176 & 1.338 & 0.007 & 0.01 & 0.023 & 1.0 & 2.45 \\
\hline 8. ul ojmstur & 1.911 & 2.078 & 1.886 & 0.033 & 1.377 & 0.200 & 1.29 & 1.24 \\
\hline 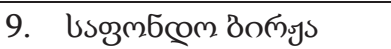 & 1.159 & 1.15 & 1.16 & 0 & 0. & 0 & $(1.50)^{*}$ & $(1.32)^{*}$ \\
\hline 10. одэœо L & 4.634 & 4.875 & 2.7451 & -0.188 & -0.543 & -0.336 & $(0.73)^{*}$ & $1.54^{*}$ \\
\hline
\end{tabular}

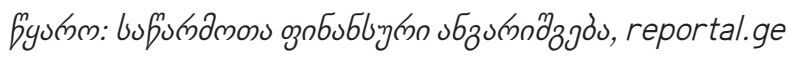




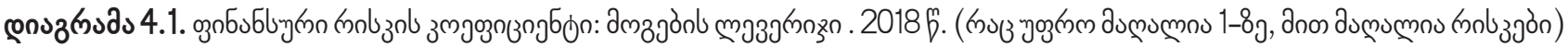

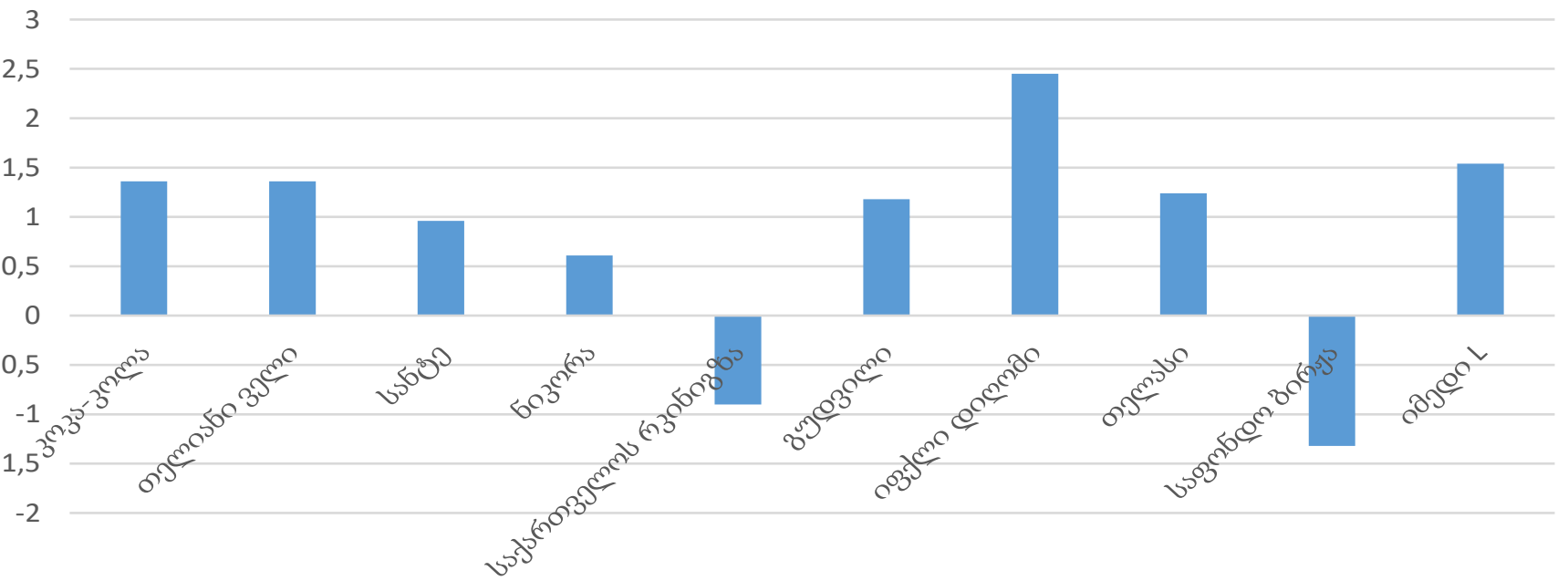

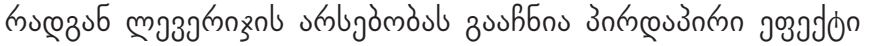

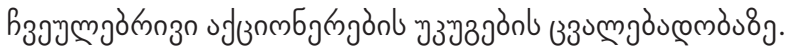

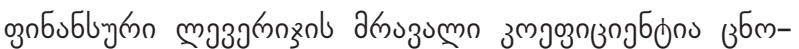

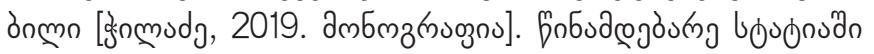

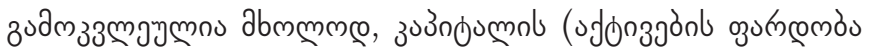

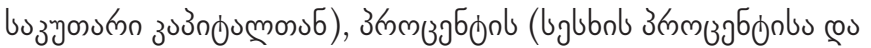

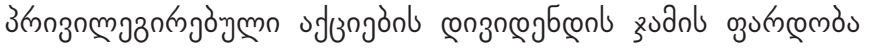

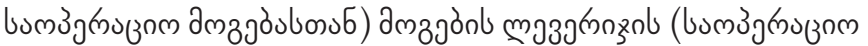

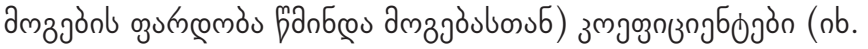

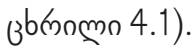

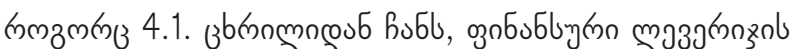

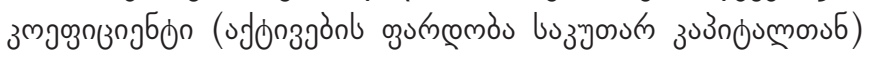

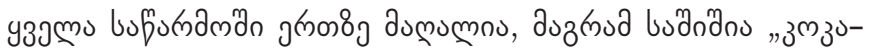

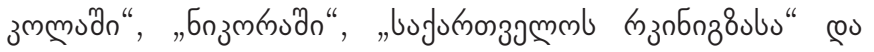

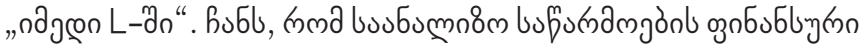

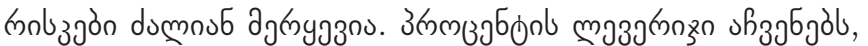

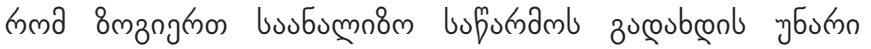

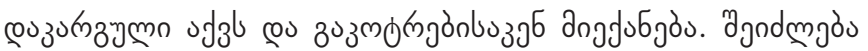

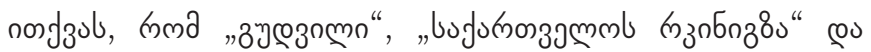

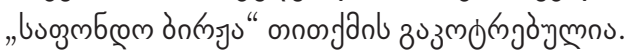

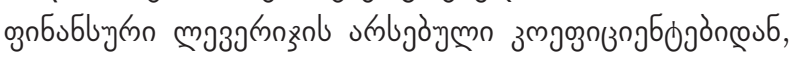

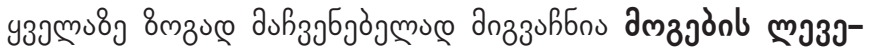

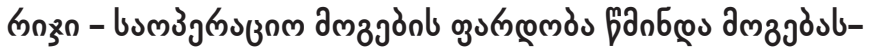

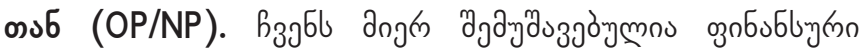

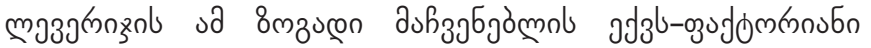

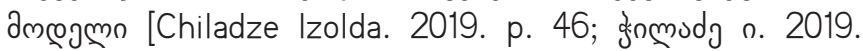
дmbmzinuogns. 33.84]:

\section{$\frac{O P}{N P}=\frac{O P}{S} * \frac{S}{P S} * \frac{P S}{A} * \frac{A}{O w C} * \frac{O w C}{P} * \frac{P}{N P} \quad \operatorname{sscos}(3)$}

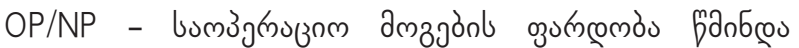
amzjöslosob.

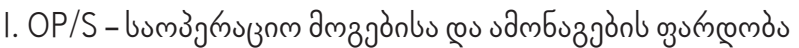
- nos yogrnm augumou glo molizgon.

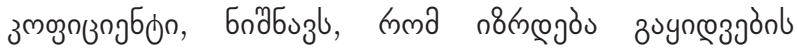

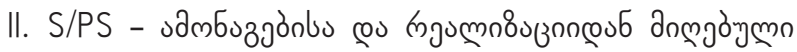

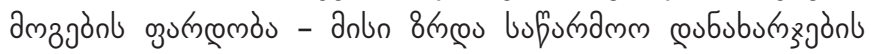

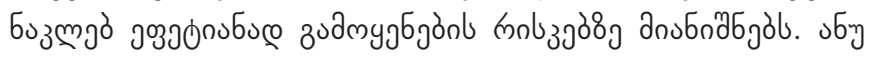

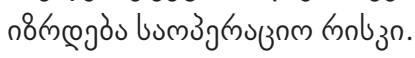

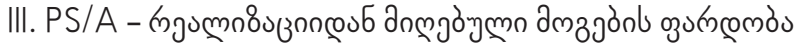

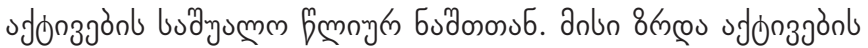

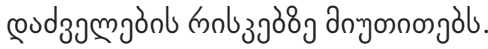

IV. A/OWC - ofonzjönl budyumm frymon budonol

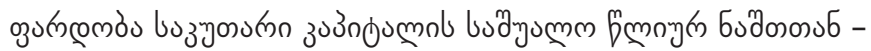

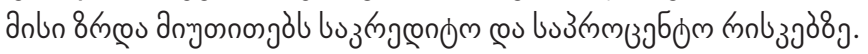

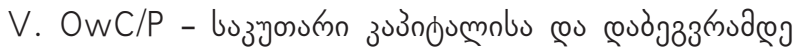

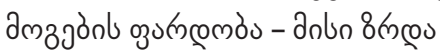

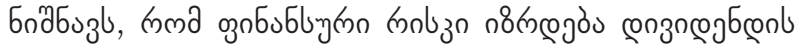

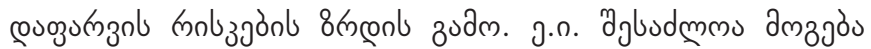

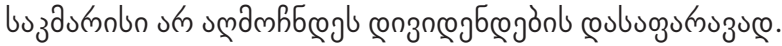

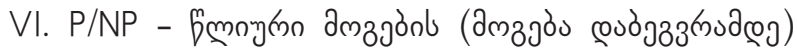

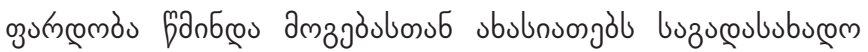

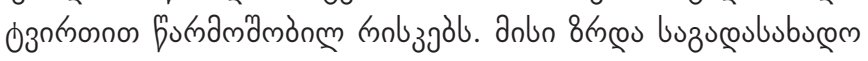

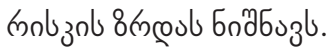

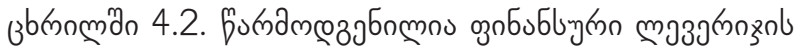

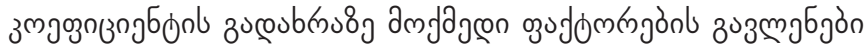

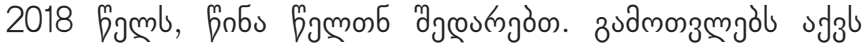
agacozo bubj.

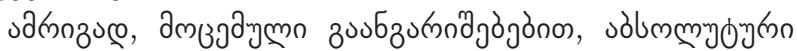

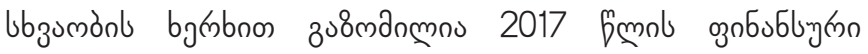

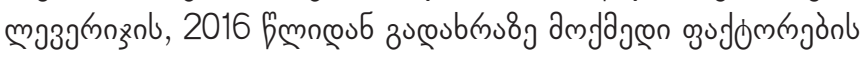

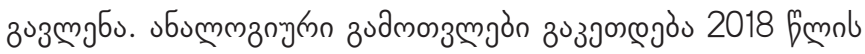

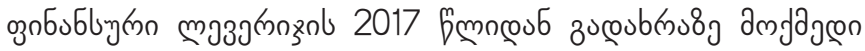

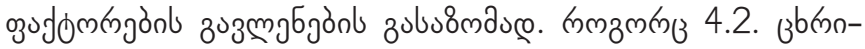

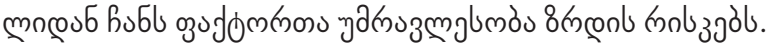

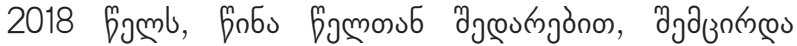

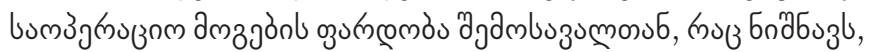

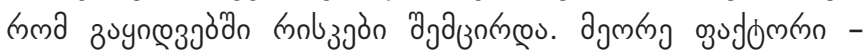




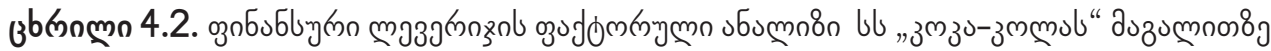

\begin{tabular}{|c|c|c|c|c|c|}
\hline \multirow[b]{2}{*}{ 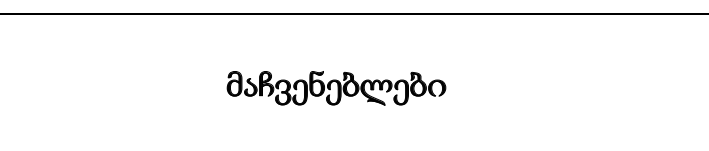 } & \multirow[b]{2}{*}{2016} & \multicolumn{2}{|c|}{2017} & \multicolumn{2}{|c|}{2018} \\
\hline & & 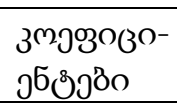 & $\begin{array}{l}\text { 8scosbms } \\
20166 \text {-@sб }\end{array}$ & 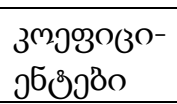 & $\begin{array}{l}\text { 8ucosbms } \\
2017 \text { 6-@s6 }\end{array}$ \\
\hline $\mathrm{s}$ & 1 & 2 & 3 & 4 & 5 \\
\hline 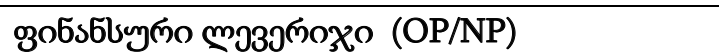 & 0.71 & 2.90 & +2.19 & 1.36 & -1.54 \\
\hline 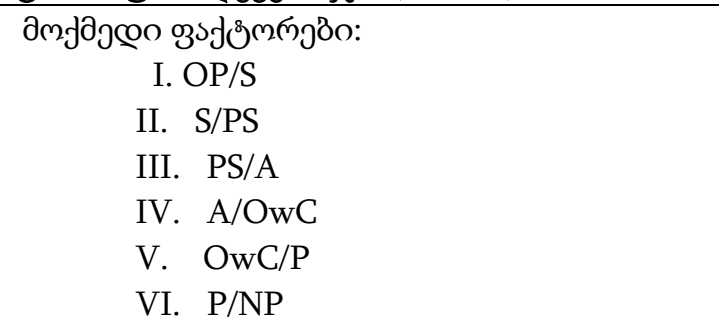 & \begin{tabular}{l}
\multicolumn{1}{c}{-} \\
0.0936 \\
3.1657 \\
0.2177 \\
2.7806 \\
3.9474 \\
1.0
\end{tabular} & $\begin{array}{l}0.1323 \\
2.9998 \\
0.2403 \\
2.1290 \\
10.4919 \\
1.3607\end{array}$ & $\begin{array}{c}- \\
+0.0387 \\
-0.1659 \\
+0.0226 \\
-0.6516 \\
+6.5445 \\
+0.3607\end{array}$ & $\begin{array}{c}- \\
0.1318 \\
3.1714 \\
0.2441 \\
2.3670 \\
5.1042 \\
1.1026\end{array}$ & 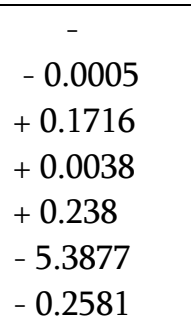 \\
\hline 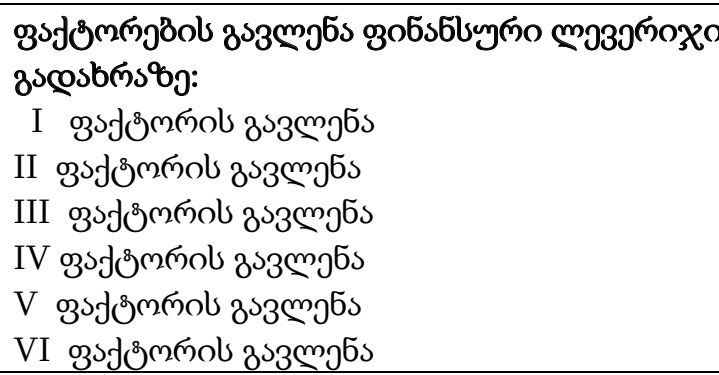 & & & $\begin{array}{c} \\
- \\
+0.2927 \\
-0.0524 \\
+0.0984 \\
-0.2453 \\
+1.3288 \\
+0.7684 \\
\end{array}$ & & $\begin{array}{c} \\
- \\
-0.011 \\
+0.1652 \\
+0.0483 \\
+0.3467 \\
-1.7705 \\
+0.3181 \\
\end{array}$ \\
\hline żäo & & & +2.19 & & -1.54 \\
\hline
\end{tabular}

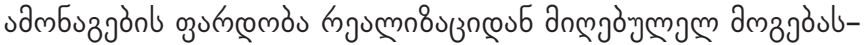

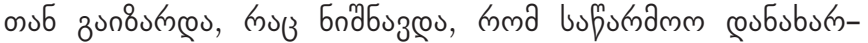

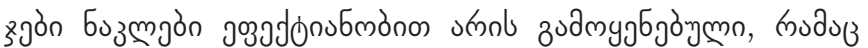

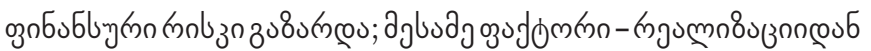

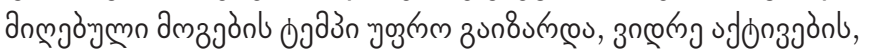

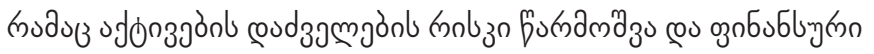

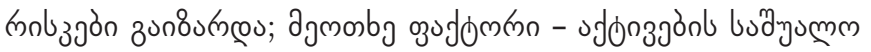

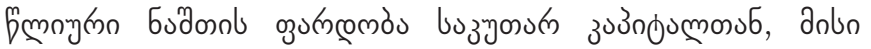

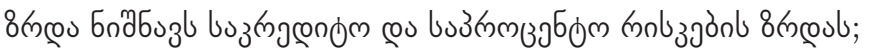

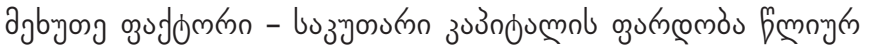

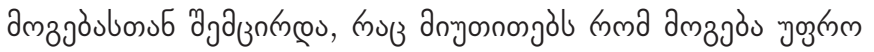

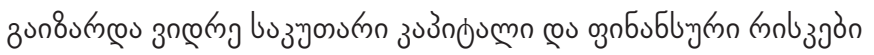

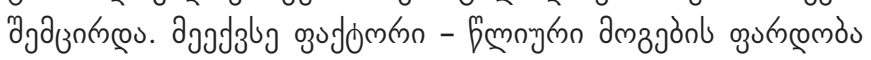

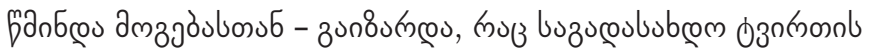

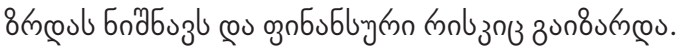

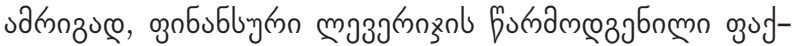

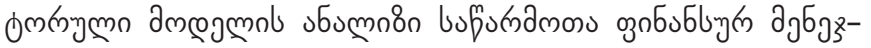

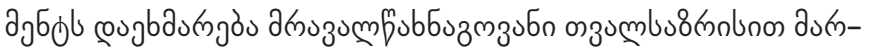

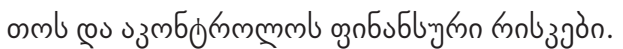

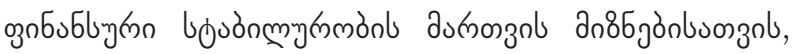

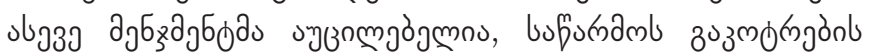

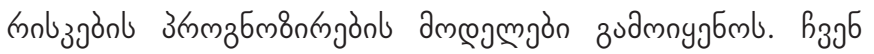

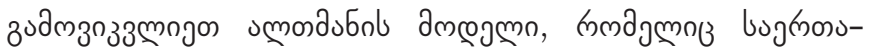

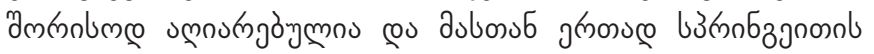

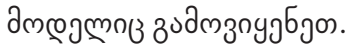

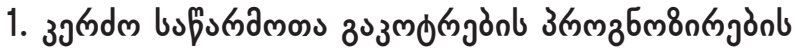

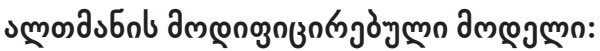

Altman Z-Ccore $=0,717 \mathrm{~A}+0,847 \mathrm{~B}+3,107 \mathrm{C}+0,42$ $D+0,998 \mathrm{E} ;$ bugous,

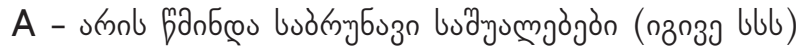

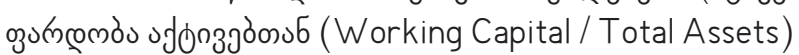

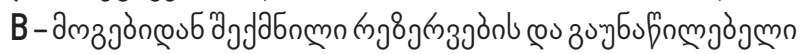

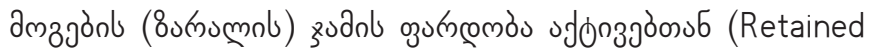
Earnings / Total Assets)

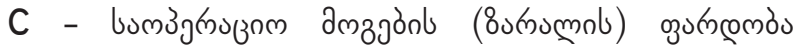
ıfonzgoosub (Earnings before Inteest and Tax / Total Assets)

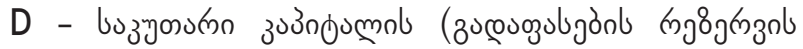

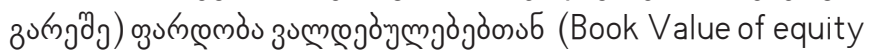
/ Total Assets)

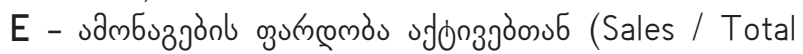
Assets)

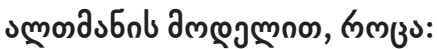

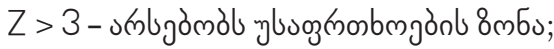

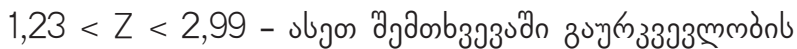

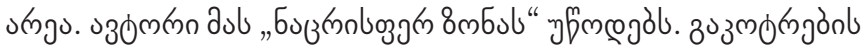

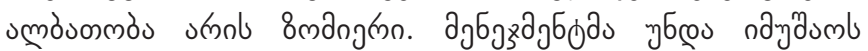

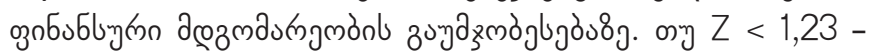

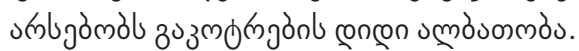

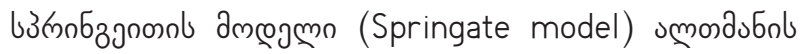

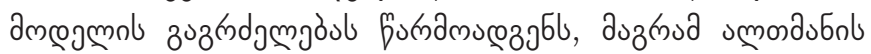

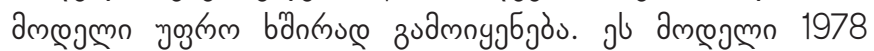

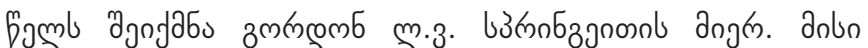

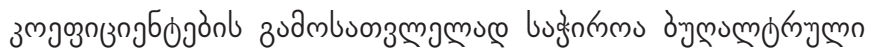

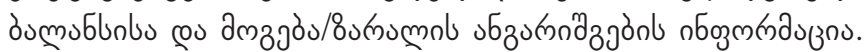




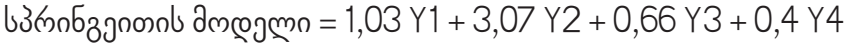

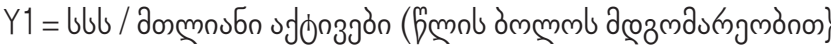

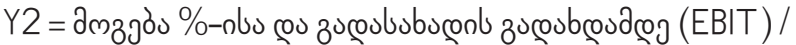
anmou

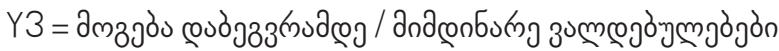
(

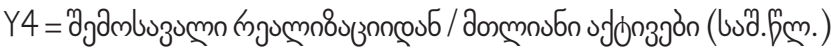

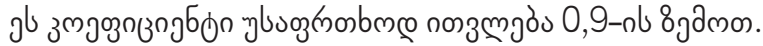

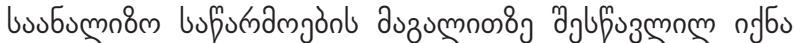

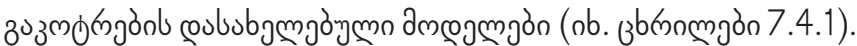

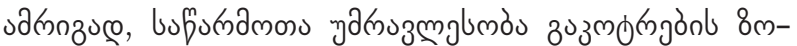

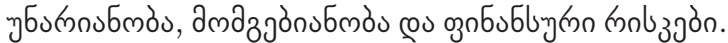

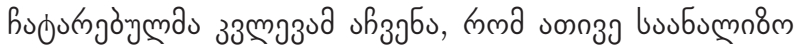

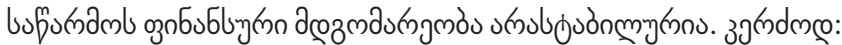

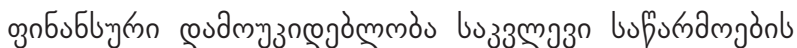

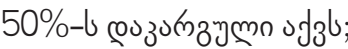

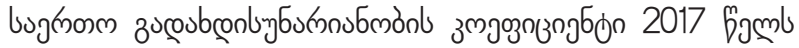

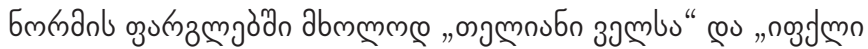

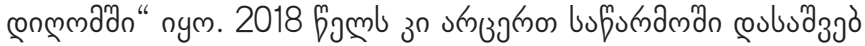

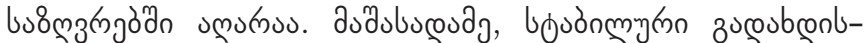

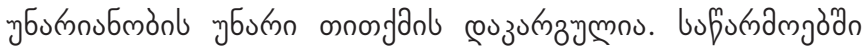

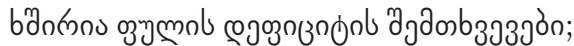

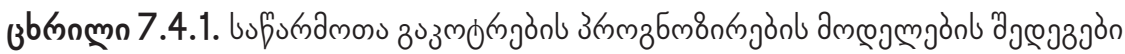

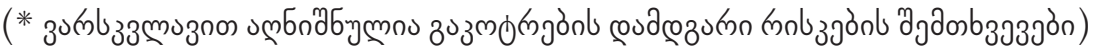

\begin{tabular}{|c|c|c|c|c|c|c|}
\hline \multirow[b]{2}{*}{ 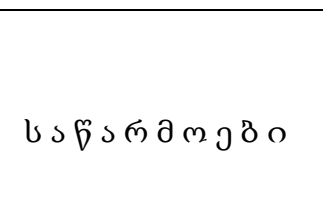 } & \multicolumn{2}{|c|}{2016} & \multicolumn{2}{|c|}{2017} & \multicolumn{2}{|l|}{2018} \\
\hline & $\begin{array}{c}\text { sलmasto } \\
\text { ylsogmonbms } \\
\text { Z > } 3\end{array}$ & 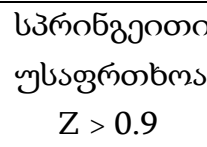 & 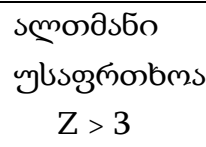 & 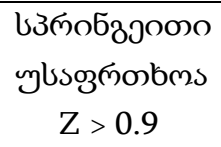 & 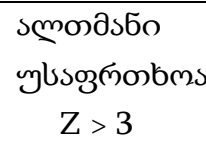 & 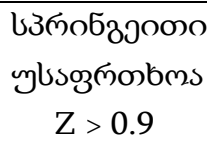 \\
\hline 1. ", $3^{\mathrm{m}} 3^{s-} 3^{\mathrm{m}} \mathrm{ms} "$ & $2.0771^{*}$ & $0.2578^{*}$ & $1.6516^{*}$ & $0.6963^{*}$ & $1.7288^{*}$ & $0.886^{*}$ \\
\hline 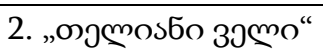 & $1.6987^{*}$ & $0.994^{*}$ & $2.8927^{*}$ & $0.7758^{*}$ & $2.6294^{*}$ & $0.5969^{*}$ \\
\hline 3. „,us6తీయ“ & $1.1818^{*}$ & 1.5141 & 4.0188 & 1.8508 & 3.648 & 1.3073 \\
\hline 4. „бозмпь“ & $2.4489^{*}$ & $0.7515^{*}$ & $2.7342^{*}$ & 1.0805 & $0.8651^{*}$ & $0.2318^{*}$ \\
\hline 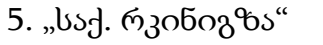 & $0.8863^{*}$ & $0.4071^{*}$ & $0.8588^{*}$ & $(1.3847) *$ & $(0.8090) *$ & $(2.0621)^{*}$ \\
\hline 6. „вуюзомо“ & $2.2262 *$ & $0.5267^{*}$ & 4.0945 & 1.1445 & 3.9964 & 2.0433 \\
\hline 7. „обдем фомман“ & 12.608 & 11.4403 & 5.4118 & 6.8746 & 7.7564 & 5.1532 \\
\hline 8. „のगmsbo“ & $2.1407^{*}$ & $1.0158^{*}$ & $1.5171^{*}$ & $0.8037^{*}$ & $2.0487^{*}$ & $0.8667^{*}$ \\
\hline 9. „иsозмбесм домыјs“ & $1.6089^{*}$ & 0.8981 & 4.7827 & $0.7526^{*}$ & $(0.2396)^{*}$ & $(0.3177) *$ \\
\hline 10. „одущо L“ & $2.6776^{*}$ & $0.0929^{*}$ & $1.6844^{*}$ & 0.9616 & $1.8996^{*}$ & $0.8837^{*}$ \\
\hline
\end{tabular}

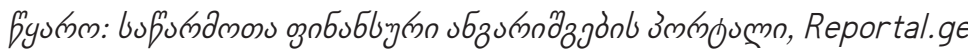

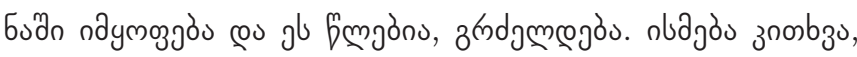
nul jмmलœ

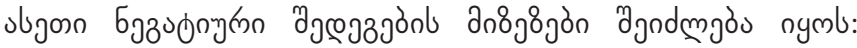

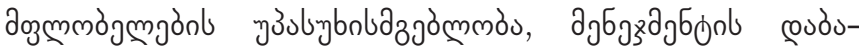
mn उஙnmog

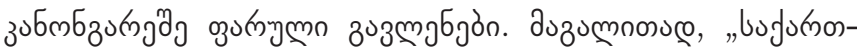

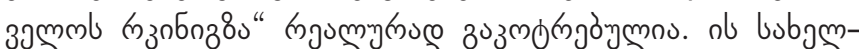

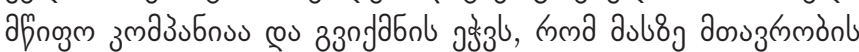

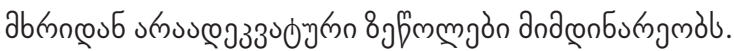

\section{csb335s}

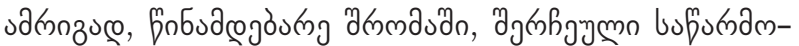

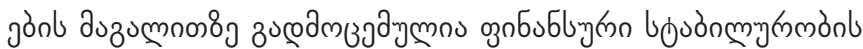

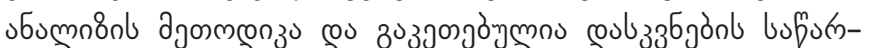

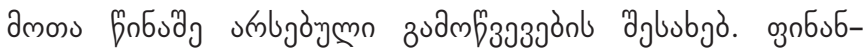

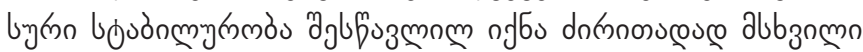

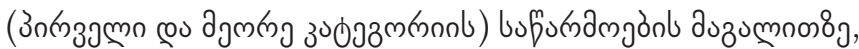
ә дам

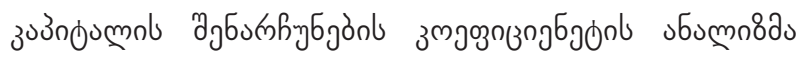

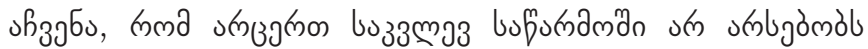
aes

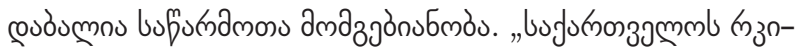

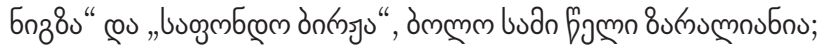

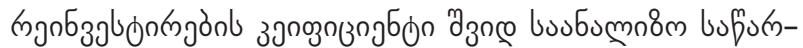

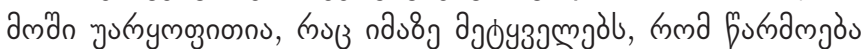

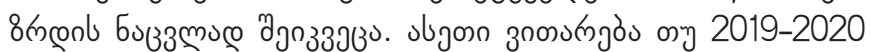

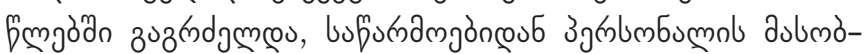

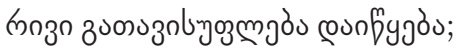

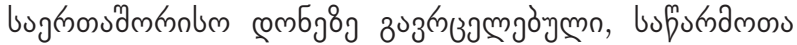

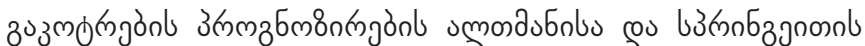

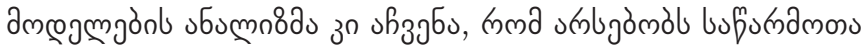

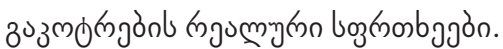

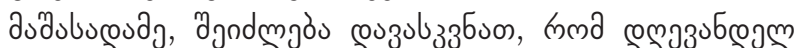

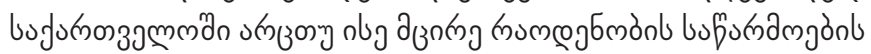

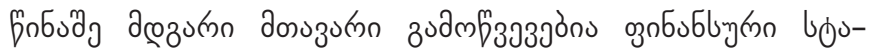

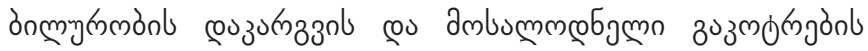
buoghonbjgòn; 


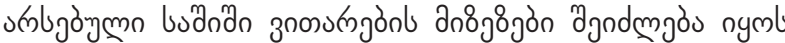

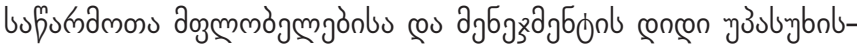

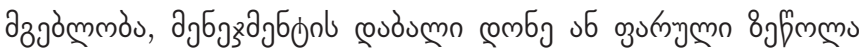

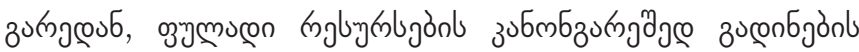

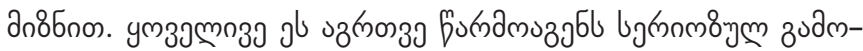

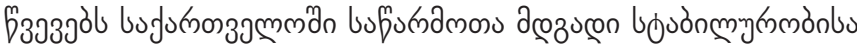

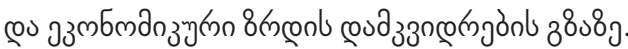

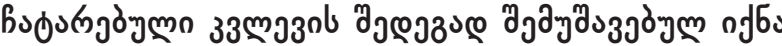

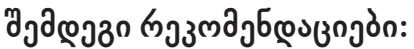

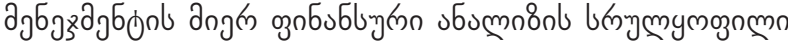

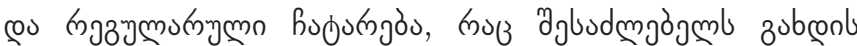

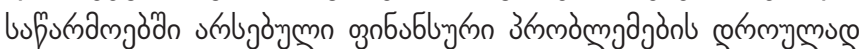

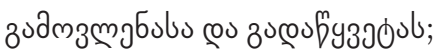

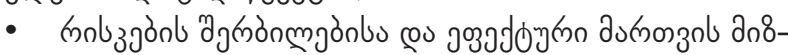

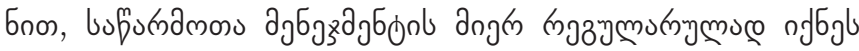

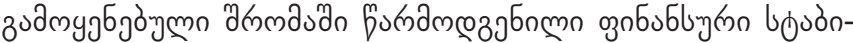

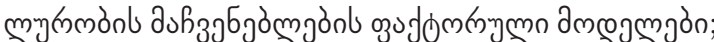

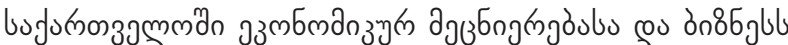

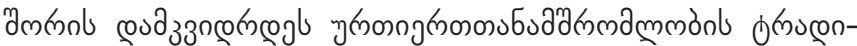

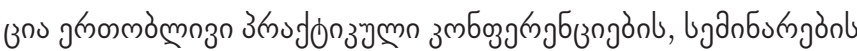

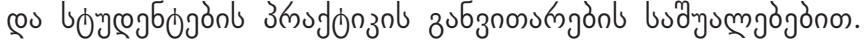

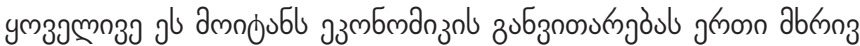

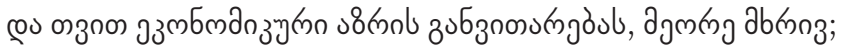

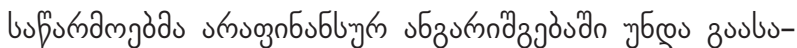

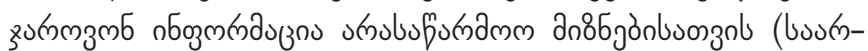

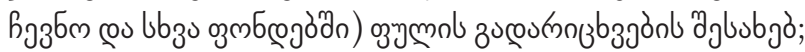

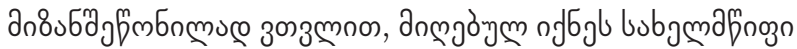
з उ

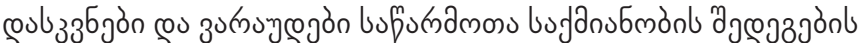

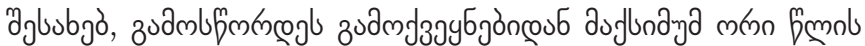

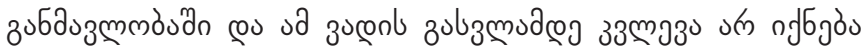

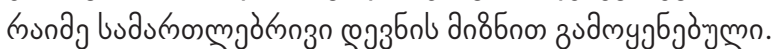

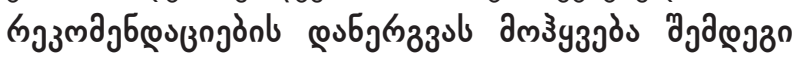

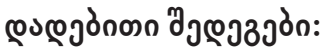

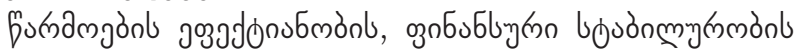

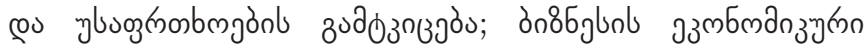

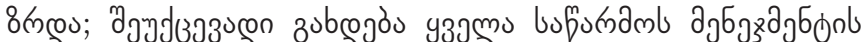

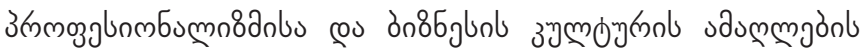
उஙmmugln; an

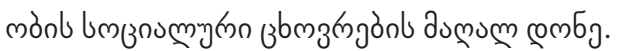

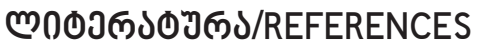

Altman Z Ccore Model Guide. (1968). https://www.cleverism.com/altman-z-score-model-guide-examples/

Brigman, Eugene F., Fundamentals of financial Manajemnt, 2011

Chiladze, I. (2017). Factor Analysis Aspects of the Enterprise's Operating Leverage. doi:10.11114/afa.v3i1. 2050

Chiladze, I. (2018).Theoretical and Practical Aspects of Profitability Factorial Analysis. $11^{\text {th }}$ International Scientific Conference: Accounting and Finance: Science, Business and Public Sector Partnership. and Studies of Accounting and Finance: Problems and Perspectives, Volume 12, Number 1, pp. 12-18. DOI:https://doi.org/10.15544/ssaf.2018.02 http://erd. asu.lt/ssaf/article/view/269/215

Chiladze Izolda (2019), Factorial Analysis of the Financial Leverage of the Enterprise, Journal: Applied Finance and Accounting Vol. 5, No. 1, February 2019 ISSN 2374-2410E-ISSN 2374-2429 Published by Redfame Publishing URL: pp. 42-48. http:// afa.redfame.com doi:10.11114/afa.v

Chiladze Izolda (2020), Improvement of the Method for the Enterprise`s Leverage Analysis. (in Georgian). Monografia. gamomcemloba „mwignobary“. https://www.tsu.ge/data/file_db/economist_faculty/leverij.pdf DuPont model. https:// en.wikipedia.org/wiki/DuPont_analysis

Garry J. Schinasi (2004) Defining Financial Stability. https://www.imf.org/external/pubs/ft/wp/2004/wp04187.pdf

Ghosh, D. K., Prakashb, A. J.,\& Ghosh, D. (2010). Leverage and Asset Allocation under Capital Market Distortion. International Journal of Business, 459-471.

http://www.craig.csufresno.edu/ijb/Volumes/Volume\%2015/V154-7.pdf

Ivo, W. (2014).Corporate Finance (3td Edition), Prentice Hall.http://www.afajof.org/SpringboardWebApp/userfiles/afa/file/ Free\%20Textbooks/Welch-ed1.pdf

Jacek Pera (2917). An enterprise's financial stability and its sustainable growth. A risk-based Perspective. file:///C:/Users/G\%20 comp\%20service/Desktop/1196-3937-1-PB.pdf

James C., Van Horn, J., \&Wachowicz, M.(2008). Fundamentals of Financial Management. Thirteenth edition, Prentice Hall. https://b-ok.asia/book/1218272/03fb95? regionChanged=\&redirect=2102656

Jonathan RerK, Peter Denarzo, Corporate Finance, 2013

Kheder, A. (2012) Operating leverage and systematic risk, Journal of business Management, 6(3), 1095-1099.http://www. academicjournals.org/article/article1380703058_Alaghi.pdf

Sanjay B. (2011).Operating Leverage and Degree of Operating Leverage.http://www.investopedia.com/terms/d/ degreeofoperatingleverage.asp Springate Score Model. (1978). https://ycharts.com/glossary/terms/springate_score.

Subramanyam, K. R.,\&Joan J. Wild, (2009). Financial Statement Analys (10td Edition). https://madnanarshad.files.wordpress. com/2014/02/fsa-by-john-j-wild-10th-wdition.pdf 